JOURNAL OF THE

AMERICAN MATHEMATICAL SOCIETY

Volume 22, Number 4, October 2009, Pages 1055-1091

S 0894-0347(09)00632-8

Article electronically published on March 24, 2009

\title{
QUANTUM COHOMOLOGY OF THE HILBERT SCHEME OF POINTS ON $\mathcal{A}_{n}$-RESOLUTIONS
}

\author{
DAVESH MAULIK AND ALEXEI OBLOMKOV
}

\section{INTRODUCTION}

1.1. Overview. Given a quasiprojective surface $S$, the Hilbert scheme $\operatorname{Hilb}_{m}(S)$ of $m$ points on $S$ parametrizes zero-dimensional subschemes of $S$ of length $m$. The Hilbert scheme is a nonsingular irreducible quasiprojective algebraic variety of dimension $2 \mathrm{~m}$. It contains an open dense set parametrizing configuration of $m$ distinct points and can be viewed as a crepant resolution of the symmetric product of $S$. The classical cohomology of these varieties has been well studied [N1, Gr, QW] and, as we shall explain later, admits a description in terms of the representation theory of Heisenberg algebras.

In this paper, we consider the following family of surfaces. Let $\zeta$ be a primitive $(n+1)$-th root of unity and let the generator of the cyclic group $\mathbb{Z}_{n+1}$ act on $\mathbb{C}^{2}$ by

$$
\left(z_{1}, z_{2}\right) \mapsto\left(\zeta z_{1}, \zeta^{-1} z_{2}\right) .
$$

We denote by $\mathcal{A}_{n}$ the minimal resolution of the quotient

$$
\mathcal{A}_{n} \rightarrow \mathbb{C}^{2} / \mathbb{Z}_{n+1}
$$

The diagonal action of $T=\left(\mathbb{C}^{*}\right)^{2}$ on $\mathbb{C}^{2}$ commutes with the cyclic group action and therefore lifts to a $T$-action on both $\mathcal{A}_{n}$ and $\operatorname{Hilb}\left(\mathcal{A}_{n}\right)$.

Our goal is to study the small quantum product on the $T$-equivariant cohomology of $\operatorname{Hilb}_{m}\left(\mathcal{A}_{n}\right)$ for all $m$ and $n$. Quantum cohomology is a deformation of the classical cohomology ring of $\operatorname{Hilb}\left(\mathcal{A}_{n}\right)$. The structure constants of the ring are defined by a virtual count of rational curves passing through specified subvarieties of the Hilbert scheme, weighted by degree.

The main theorem of this paper is an explicit operator formula for quantum multiplication by divisors in $H_{T}^{2}\left(\operatorname{Hilb}_{m}\left(\mathcal{A}_{n}\right), \mathbb{Q}\right)$. These operators have a simple expression in terms of the action of the affine Lie algebra $\widehat{\mathfrak{g l}}(n+1)$ on its basic representation. Under the assumption of a nondegeneracy conjecture (see Section 6.3), the divisor operators generate the entire quantum cohomology ring and, in particular, can be used to calculate the full genus 0 Gromov-Witten theory.

In the case of $\mathbb{C}^{2}$, the quantum cohomology ring has been completely calculated in OP1. Along with our results here, these surfaces are the only surfaces for

Received by the editors March 5, 2008.

2000 Mathematics Subject Classification. Primary 14N35.

Key words and phrases. Hilbert scheme of points, quantum cohomology.

The first author was partially supported by an NSF Graduate Fellowship and a Clay Research Fellowship.

The second author was partially supported by NSF grants DMS-0111298 and DMS-0701387.

(C)2009 American Mathematical Society

Reverts to public domain 28 years from publication 
which the divisor operators have been fully calculated for an arbitrary number of points. Our strategy is motivated by the approach of OP1 for $\mathbb{C}^{2}$. The presence of compact directions on the underlying surfaces greatly complicates the geometry of the Hilbert scheme; the existence of a holomorphic symplectic form is essential for circumventing these difficulties.

1.2. Relation to other theories. Using the results of $[\mathrm{M}], \mathrm{MO}]$, we show that the divisor operators of this paper satisfy a triangle of equivalences between the Gromov-Witten theory of $\mathcal{A}_{n} \times \mathbf{P}^{1}$, the Donaldson-Thomas theory of $\mathcal{A}_{n} \times \mathbf{P}^{1}$, and the quantum cohomology of the Hilbert scheme of points on the $\mathcal{A}_{n}$ surface.

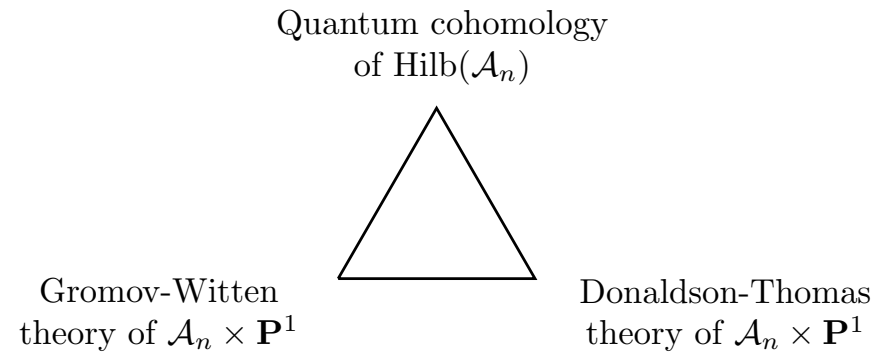

The above triangle was first shown to hold for $\mathbf{C}^{2}$ in $[\mathrm{BP}$, OP1], OP2]. While the equivalence between Gromov-Witten theory and Donaldson-Thomas theory is expected to hold for arbitrary threefolds, the relationship with the quantum cohomology of the Hilbert scheme breaks down for a general surface, at least in the specific form we describe here. Our work for $\mathcal{A}_{n}$ surfaces provides the only other examples for which this triangle is known to hold.

\section{Statement of theorems}

In this section, we state precisely the operators for multiplication by divisors on the quantum cohomology ring. In order to express these cleanly, we explain how to write the cohomology of $\operatorname{Hilb}\left(\mathcal{A}_{n}\right)$ in terms of the representation theory of $\widehat{\mathfrak{g l}}(n+1)$.

2.1. Topology of $\mathcal{A}_{n}$ and the root lattice. We first set notation for the geometry of the $\mathcal{A}_{n}$ surfaces. Viewed as a crepant resolution of a quotient singularity, the exceptional locus of $\mathcal{A}_{n}$ consists of a chain of $n$ rational curves $E_{1}, \ldots, E_{n}$ with intersection matrix given by the negative Cartan matrix for the Dynkin diagram $A_{n}$. That is, each $E_{i}$ has self-intersection -2 and intersects $E_{i-1}$ and $E_{i+1}$ transversely. These classes span $H^{2}\left(\mathcal{A}_{n}, \mathbb{Q}\right)$ and, along with the identity class, span the full cohomology ring of $\mathcal{A}_{n}$. We will also work with the dual basis $\left\{\omega_{1}, \ldots, \omega_{n}\right\}$ of $H^{2}\left(\mathcal{A}_{n}, \mathbb{Q}\right)$, defined by the property that

$$
\left\langle\omega_{i}, E_{j}\right\rangle=\delta_{i, j}
$$

under the Poincaré pairing.

Under the $T$-action, there are $n+1$ fixed points $p_{1}, \ldots, p_{n+1}$; the tangent weights at the fixed point $p_{i}$ are given by

$$
\begin{gathered}
w_{i}^{L}:=(n+2-i) t_{1}+(1-i) t_{2}, \\
w_{i}^{R}:=(-n+i-1) t_{1}+i t_{2} .
\end{gathered}
$$


The $E_{i}$ are the $T$-fixed curves joining $p_{i}$ to $p_{i+1}$. We denote by $E_{0}$ and $E_{n+1}$ the noncompact $T$-fixed curve direction at $p_{1}$ and $p_{n+1}$, respectively.

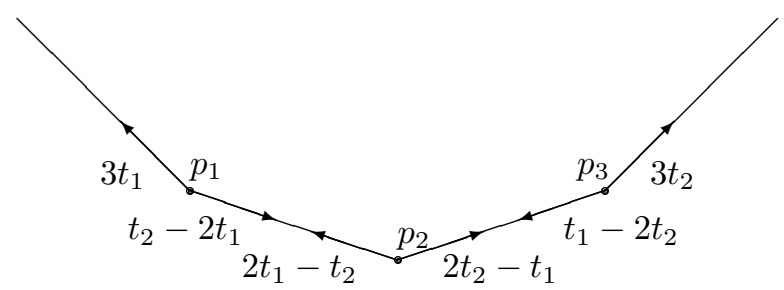

As usual, we denote by $\mathfrak{g l}(n+1)$ the Lie algebra (defined over $\mathbb{Q})$ of $(n+1) \times(n+1)$ matrices. We will denote by $e_{i j} \in \mathfrak{g l}(n+1)$ the matrix with 1 in position $(i, j)$ and 0 everywhere else. With respect to the Cartan subalgebra $\mathfrak{h}$ of diagonal matrices, the roots are given by functionals $\alpha_{i j} \in \mathfrak{h}^{*}$ which take the value $a_{i i}-a_{j j}$ on the diagonal matrix $\left(a_{k k}\right)$. There is an identification of lattices between $H_{2}\left(\mathcal{A}_{n}, \mathbb{Z}\right)$ with the $A_{n}$ root lattice obtained by sending the exceptional curves $E_{i}$ to the simple roots $\alpha_{i, i+1}$. Under this identification, there is a distinguished set of effective curve classes

$$
\alpha_{i j}=E_{i}+\cdots+E_{j-1}
$$

which correspond to positive roots in the $A_{n}$ lattice.

2.2. Fock space formalism. In this section, we introduce the Fock space modelled on $H_{T}^{*}\left(\mathcal{A}_{n}, \mathbb{Q}\right)$ and explain the identification with $H_{T}^{*}\left(\operatorname{Hilb}\left(\mathcal{A}_{n}\right), \mathbb{Q}\right)$. These constructions for projective surfaces were first provided in [N1, Gr] and extended in this setting to the equivariant context in $[\mathrm{QW}$. Consider the Heisenberg algebra $\mathcal{H}$ generated over the field $\mathbb{Q}\left(t_{1}, t_{2}\right)$ by a central element $c$ and elements

$$
\mathfrak{p}_{k}(\gamma), \gamma \in H_{T}^{*}\left(\mathcal{A}_{n}, \mathbb{Q}\right), k \in \mathbb{Z}, k \neq 0,
$$

so that $\mathfrak{p}_{k}(\gamma)$ are $\mathbb{Q}\left(t_{1}, t_{2}\right)$-linear in the labels $\gamma$. The Lie algebra structure on $\mathcal{H}$ is defined by the following commutation relations:

$$
\begin{gathered}
{\left[\mathfrak{p}_{k}\left(\gamma_{1}\right), \mathfrak{p}_{l}\left(\gamma_{2}\right)\right]=-k \delta_{k+l}\left\langle\gamma_{1}, \gamma_{2}\right\rangle \cdot c,} \\
{\left[c, \mathfrak{p}_{k}(\gamma)\right]=0 .}
\end{gathered}
$$

Our sign convention for the commutator differs from $\mathrm{N1}$ but simplifies later formulas. Notice that we can pick a basis of cohomology for which the Poincaré pairing never takes denominators that are divisible by $\left(t_{1}+t_{2}\right)$; as a result, $\mathcal{H}$ can be defined over the ring

$$
R=\mathbb{Q}\left[t_{1}, t_{2}\right]_{\left(t_{1}+t_{2}\right)}
$$

of rational functions with nonnegative valuation at $\left(t_{1}+t_{2}\right)$.

The Fock space $\mathcal{F}_{\mathcal{A}_{n}}$ is freely generated over the ring $\mathbb{Q}\left[t_{1}, t_{2}\right]$ by the action of the commuting creation operators $\mathfrak{p}_{-k}(\gamma)$ for $k>0$ and $\gamma \in H_{T}^{*}\left(\mathcal{A}_{n}, \mathbb{Q}\right)$ on the vacuum vector $v_{\emptyset}$. There is an orthogonal grading

$$
\mathcal{F}_{\mathcal{A}_{n}}=\bigoplus_{m \geq 0} \mathcal{F}_{\mathcal{A}_{n}}^{(m)}
$$


induced by defining the degree of $v_{\emptyset}$ to be zero and the degree of each operator $\mathfrak{p}_{k}(\gamma)$ to be $-k$.

After an extension of scalars to the fraction field $\mathbb{Q}\left(t_{1}, t_{2}\right), \mathcal{F}_{\mathcal{A}_{n}}$ admits the structure of a representation of $\mathcal{H}$. Under this action, the annihiliation operators $\mathfrak{p}_{k}(\gamma)$ with $k>0$ kill the vacuum vector and the central element $c$ acts trivially. Similarly, we define a nondegenerate pairing on $\mathcal{F}_{\mathcal{A}_{n}} \otimes \mathbb{Q}\left(t_{1}, t_{2}\right)$ by requiring

$$
\left\langle v_{\emptyset} \mid v_{\emptyset}\right\rangle=1
$$

and specifiying the adjoint

$$
\mathfrak{p}_{k}(\gamma)^{*}=(-1)^{k} \mathfrak{p}_{-k}(\gamma) .
$$

There is a graded isomorphism

$$
\mathcal{F}_{\mathcal{A}_{n}}=\bigoplus_{m \geq 0} H_{T}^{*}\left(\operatorname{Hilb}_{m}\left(\mathcal{A}_{n}\right), \mathbb{Q}\right) .
$$

Under this isomorphism, the Heisenberg operators $\mathfrak{p}_{k}(\gamma)$ are defined by correspondences between Hilbert schemes of different numbers of points. The inner product on $\mathcal{F}_{\mathcal{A}_{n}}$ over $\mathbb{Q}\left(t_{1}, t_{2}\right)$ defined above corresponds to the Poincaré pairing on $\operatorname{Hilb}_{m}\left(\mathcal{A}_{n}\right)$ defined by $T$-equivariant residue.

2.3. Nakajima basis. If we work with a fixed basis $\left\{\gamma_{0}, \ldots, \gamma_{n}\right\}$ of $H_{T}^{*}\left(\mathcal{A}_{n}, \mathbb{Q}\right)$, our Fock space has a natural basis indexed as follows. Given a nonnegative integer $m$, a cohomology-weighted partition of $m$ consists of an unordered set of pairs

$$
\vec{\mu}=\left\{\left(\mu^{(1)}, \gamma_{i_{1}}\right), \ldots,\left(\mu^{(l)}, \gamma_{i}^{l}\right)\right\},
$$

where $\left\{\mu^{(1)}, \ldots, \mu^{(l)}\right\}$ is a partition whose parts are labelled by elements $\gamma_{i_{k}}$ in our basis. For each cohomology-weighted partition $\vec{\mu}$ as above, the associated basis element is given by

$$
\frac{1}{\mathfrak{z}(\vec{\mu})} \prod_{k=1}^{l} \mathfrak{p}_{-\mu^{(k)}}\left(\gamma_{i_{k}}\right) v_{\emptyset},
$$

where

$$
\mathfrak{z}(\vec{\mu})=\prod \mu^{(k)} \cdot|\operatorname{Aut}(\vec{\mu})| .
$$

We will also denote this basis element by $\vec{\mu}$ when there is no confusion. The associated basis of $H_{T}^{*}\left(\operatorname{Hilb}_{m}\left(\mathcal{A}_{n}\right), \mathbb{Q}\right)$ will be called the Nakajima basis. It is clear from the definition that this basis respects the grading of $\mathcal{F}_{\mathcal{A}_{n}}$ so that cohomologyweighted partitions of $m$ form a basis of $H_{T}^{*}\left(\operatorname{Hilb}_{m}\left(\mathcal{A}_{n}\right), \mathbb{Q}\right)$ over $\mathbb{Q}\left[t_{1}, t_{2}\right]$. If we want to stress the underlying choice of basis $\gamma_{k}$, we will write

$$
\mu_{1}\left(\gamma_{1}\right) \cdot \mu_{2}\left(\gamma_{2}\right) \ldots \mu_{r}\left(\gamma_{r}\right)
$$

where $\mu_{k}$ is the subpartition of $\mu$ labelled with $\gamma_{k}$.

The cohomological degree of a basis element $\vec{\mu}$ under these identifications is

$$
2(m-l(\mu))+\sum \operatorname{deg}\left(\gamma_{i_{k}}\right) .
$$

Finally, under the $T$-equivariant Poincaré pairing, the dual basis of the Nakajima basis is given, up to constant factors, by cohomology-weighted partitions labelled with the dual basis of $\left\{\gamma_{i}\right\}$. 
In terms of the basis $\left\{1, \omega_{1}, \ldots, \omega_{n}\right\}$, we can see from the formula for cohomological degree that there are two types of divisors in the Nakajima basis. First, we have

$$
D=-\left\{(2,1),(1,1)^{m-2}\right\}
$$

which is proportional to the boundary divisor on $\operatorname{Hilb}_{m}\left(\mathcal{A}_{n}\right)$ where two points collide. Second, we have for $i=1, \ldots, n$,

$$
\left(1, \omega_{i}\right)=\left\{\left(1, \omega_{i}\right),(1,1)^{m-1}\right\} .
$$

These latter divisors are clearly nonnegative on effective curve classes. We will give explicit formulas for quantum multiplication by these elements. We will also use this basis of divisors to measure degrees of curve classes.

2.4. Affine algebra $\widehat{\mathfrak{g l}}(n+1)$ and the basic representation. The Fock space description given above can be reinterpreted in terms of the representation theory of the affine algebra $\widehat{\mathfrak{g l}}(n+1)$. While the previous discussion is valid for any surface $S$, this interpretation is a special feature of the $\mathcal{A}_{n}$ surfaces.

The Lie algebra $\widehat{\mathfrak{g}}=\widehat{\mathfrak{g l}}(n+1)$ is defined over $\mathbb{Q}$ in terms of a central extension of the loop algebra $\mathfrak{g l}(n+1) \otimes \mathbb{Q}\left[t, t^{-1}\right]$. It is generated by elements

$$
x(k)=x \cdot t^{k}, \quad x \in \mathfrak{g l}(n+1), \quad k \in \mathbb{Z},
$$

a central element $c$, and a differential $d$. The defining relations are

$$
\begin{gathered}
{[x(k), y(l)]=[x, y](k+l)+k \delta_{k+l, 0} \operatorname{tr}(x y) c,} \\
{[d, x(k)]=k x(k), \quad[d, c]=0,}
\end{gathered}
$$

where $\operatorname{tr}(x y)$ refers to the trace of the matrix $x y$. The Cartan subalgebra of $\widehat{\mathfrak{g}}$ is given by

$$
\hat{\mathfrak{h}}=\mathfrak{h} \oplus \mathbb{Q} c \oplus \mathbb{Q} d .
$$

Using this direct sum decomposition, we can write the dual space

$$
\hat{\mathfrak{h}}^{*}=\mathfrak{h}^{*} \oplus \mathbb{Q} \Lambda \oplus \mathbb{Q} \delta,
$$

where $\Lambda(c)=1, \Lambda(d)=0$ and $\delta(d)=1, \delta(c)=0$. As in the finite-dimensional situation, there is a theory of roots and weights lying in this dual space. The roots of $\widehat{\mathfrak{g}}$ are given by

$$
\Delta=\left\{k \delta+\alpha_{i, j}, k \in \mathbb{Z}\right\} \cup\{k \delta, k \neq 0\} .
$$

Associated to the weight $\Lambda$, there is a unique irreducible highest weight representation $\left(V_{\Lambda}, \rho\right)$, containing a vector $v$ such that

$$
\rho(\mathfrak{g l}(n+1) \otimes \mathbb{Q}[t]) v=0
$$

and

$$
\rho(c) v=v
$$

This is known as the basic representation of $\widehat{\mathfrak{g}}$.

We have an embedding of Lie algebras of the Heisenberg algebra $\mathcal{H}$ associated to $\mathcal{A}_{n}$,

given by the map

$$
\mathcal{H} \rightarrow \widehat{\mathfrak{g}} \otimes \mathbb{Q}\left(t_{1}, t_{2}\right)
$$

$$
\begin{gathered}
\mathfrak{p}_{-k}(1) \mapsto \operatorname{Id}(-k), \quad \mathfrak{p}_{k}(1) \mapsto-\operatorname{Id}(k) /\left((n+1)^{2} t_{1} t_{2}\right), \quad k>0, \\
\mathfrak{p}_{k}\left(E_{i}\right) \mapsto e_{i, i}(k)-e_{i+1, i+1}(k), \quad c \mapsto 1 .
\end{gathered}
$$


This embedding is compatible with the identification of $H_{2}\left(\mathcal{A}_{n}, \mathbb{Z}\right)$ with the finite $A_{n}$ root lattice. We identify $\mathcal{H}$ with its image inside $\widehat{\mathfrak{g}} \otimes \mathbb{Q}\left(t_{1}, t_{2}\right)$.

Rather than study the full representation $V_{\Lambda}$, we will work with the subspace

$$
W=\bigoplus_{m \geq 0} V_{\Lambda}[\Lambda-m \delta]
$$

where $V[\alpha]$ denotes the weight space of $V$ associated to the weight $\alpha$. By construction, $W$ is graded by nonnegative integers. Moreover, after an extension of scalars, the space $W \otimes \mathbb{Q}\left(t_{1}, t_{2}\right)$ is preserved by the action of $\mathcal{H}$. We then have the following easy observation.

Proposition 2.1. There is an isomorphism of $\mathcal{H}$-modules

$$
W \otimes \mathbb{Q}\left(t_{1}, t_{2}\right) \rightarrow \mathcal{F}_{\mathcal{A}_{n}} \otimes \mathbb{Q}\left(t_{1}, t_{2}\right)
$$

uniquely specified by requiring that $v$ map to $v_{\emptyset}$.

Although we will not need it, Nakajima has identified the entire basic representation of $\widehat{\mathfrak{g}}$ with the cohomology of various moduli spaces. Also, note that while we have extended scalars to $\mathbb{Q}\left(t_{1}, t_{2}\right)$, all objects and maps given here are again defined over $R=\mathbb{Q}\left[t_{1}, t_{2}\right]_{\left(t_{1}+t_{2}\right)}$.

2.5. Quantum cohomology. Let $\beta \in H_{2}\left(\operatorname{Hilb}_{m}\left(\mathcal{A}_{n}\right), \mathbb{Z}\right)$ be a curve class. Given cohomology classes $\vec{\mu}_{1}, \ldots, \vec{\mu}_{k} \in H_{T}^{*}\left(\operatorname{Hilb}_{m}\left(\mathcal{A}_{n}\right), \mathbb{Q}\right)$, the $k$-point genus 0 GromovWitten invariant is defined by integration against the $T$-equivariant virtual class of the moduli space of genus 0 stable maps

$$
\left\langle\overrightarrow{\mu_{1}}, \ldots, \overrightarrow{\mu_{k}}\right\rangle_{0, k, \beta}^{\mathrm{Hilb}}=\int_{\left[\bar{M}_{0, k}\left(\operatorname{Hilb}\left(\mathcal{A}_{n}\right), \beta\right]^{\mathrm{vir}}\right.} \operatorname{ev}_{1}^{*} \overrightarrow{\mu_{1}} \ldots \mathrm{ev}_{k}^{*} \overrightarrow{\mu_{k}},
$$

where $\mathrm{ev}_{i}: \bar{M}_{0, k}\left(\operatorname{Hilb}\left(\mathcal{A}_{n}\right), \beta\right) \rightarrow \operatorname{Hilb}\left(\mathcal{A}_{n}\right)$ denote evaluation maps. We can then encode these invariants in the $k$-point generating function

$$
\left\langle\overrightarrow{\mu_{1}}, \ldots, \overrightarrow{\mu_{k}}\right\rangle^{\mathrm{Hilb}}=\sum_{\beta}\left\langle\overrightarrow{\mu_{1}}, \ldots, \overrightarrow{\mu_{k}}\right\rangle_{0, k, \beta}^{\mathrm{Hilb}} q^{D \cdot \beta} \prod_{i=1}^{n} s_{i}^{\left(1, \omega_{i}\right) \cdot \beta} \in \mathbb{Q}\left(t_{1}, t_{2}\right)((q))\left[\left[s_{1}, \ldots, s_{n}\right]\right],
$$

where again $D$ and $\left(1, \omega_{i}\right)$ are the basis of divisors on $\operatorname{Hilb}\left(\mathcal{A}_{n}\right)$ described earlier.

Since $\mathcal{A}_{n}$ is noncompact, some care is required to define the above integrals rigorously. Although $\bar{M}_{0, k}\left(\operatorname{Hilb}\left(\mathcal{A}_{n}\right), \beta\right)$ may be noncompact, its $T$-fixed locus is necessarily compact. In this case, we can define the above integral by the pushforward of its equivariant residue. In the compact case, this agrees with the original definition by the virtual localization formula.

In the case of $k=3$, we can define the quantum product on

$$
Q H_{T}\left(\operatorname{Hilb}_{m}\left(\mathcal{A}_{n}\right)\right)=H_{T}^{*}\left(\operatorname{Hilb}_{m}\left(\mathcal{A}_{n}\right), \mathbb{Q}\right) \otimes \mathbb{Q}\left(t_{1}, t_{2}\right)((q))\left[\left[s_{1}, \ldots, s_{n}\right]\right]
$$

as follows. Given $\vec{\mu}, \vec{\nu}, \vec{\rho}$, the quantum product $\circ$ is defined using the structure constants

$$
\langle\vec{\mu} \mid \vec{\rho} \circ \vec{\nu}\rangle=\langle\vec{\mu}, \vec{\rho}, \vec{\nu}\rangle^{\mathrm{Hilb}} \in \mathbb{Q}\left(t_{1}, t_{2}\right)((q))\left[\left[s_{1}, \ldots, s_{n}\right]\right] .
$$

The brackets on the left-hand side of the above equation denote the inner product on Fock space. It is a standard fact that these structure constants define a ring deformation of the classical equivariant cohomology ring. 
2.6. Operator formulas. The main object of study in this paper are the twopoint genus 0 invariants for $\operatorname{Hilb}\left(\mathcal{A}_{n}\right)$. These can be encoded in an operator on Fock space; more precisely, we define the operator $\Theta\left(q, s_{1}, \ldots, s_{n}\right)$ by the equality

$$
\left\langle\vec{\mu}\left|\Theta\left(q, s_{1}, \ldots, s_{n}\right)\right| \vec{\nu}\right\rangle=\langle\vec{\mu}, \vec{\nu}\rangle^{\text {Hilb }} .
$$

On the left-hand side, the angle brackets refer to the inner product on Fock space induced by Poincaré duality.

Consider the following function of $q, s_{1}, \ldots, s_{n}$ with coefficients in $U(\widehat{\mathfrak{g}})$ :

$$
\Omega_{+}:=\sum_{1 \leq i<j \leq n+1} \sum_{k \in \mathbb{Z}}: e_{j i}(k) e_{i j}(-k): \log \left(1-(-q)^{k} s_{i} \ldots s_{j-1}\right) .
$$

In this expression, we use the normal ordering shorthand where

$$
: e_{j i}(k) e_{i j}(-k):= \begin{cases}e_{j i}(k) e_{i j}(-k), & k<0 \text { or } k=0, i<j, \\ e_{i j}(-k) e_{j i}(k), & \text { otherwise. }\end{cases}
$$

Moreover, we expand the logarithms so that the Taylor expansion has nonnegative exponents in the $s$ variables.

A priori, the above expression defines an operator on the entire basic representation $V_{\Lambda}$. However, a direct calculation shows that each summand $: e_{i j}(k) e_{j i}(-k)$ : commutes with elements of the Cartan subalgebra $\hat{\mathfrak{h}}$. As a result, $\Omega_{+}$preserves each weight subspace of $V_{\Lambda}$ and in particular descends to a well-defined operator on each graded component:

$$
V_{\Lambda}[\Lambda-m \delta] \otimes \mathbb{Q}\left(t_{1}, t_{2}\right)=H_{T}^{*}\left(\operatorname{Hilb}_{m}\left(\mathcal{A}_{n}\right), \mathbb{Q}\right) .
$$

Moreover, for each graded piece, it is clear that only finitely many summands in $\Omega_{+}$contribute.

We also have the operator

$$
\Omega_{0}=-\sum_{k \geq 1}\left[(n+1) t_{1} t_{2} \mathfrak{p}_{-k}(1) \mathfrak{p}_{k}(1)+\sum_{i=1}^{n} \mathfrak{p}_{-k}\left(E_{i}\right) \mathfrak{p}_{k}\left(\omega_{i}\right)\right] \log \left(\frac{1-(-q)^{k}}{1-(-q)}\right)
$$

and the sum

$$
\Omega=\Omega_{0}+\Omega_{+} .
$$

The expression for $\Omega_{0}$ is already written in terms of Heisenberg operators, so it obviously acts on Fock space.

Our main theorem is that $\Omega\left(q, s_{1}, \ldots, s_{n}\right)$ is essentially the operator encoding two-point Gromov-Witten invariants for $\operatorname{Hilb}\left(\mathcal{A}_{n}\right)$.

Theorem 2.1. The generating function of two-point invariants of $\operatorname{Hilb}_{m}\left(\mathcal{A}_{n}\right)$ is given by the following operator equality:

$$
\Theta\left(q, s_{1}, \ldots, s_{n}\right)=\left(t_{1}+t_{2}\right) \cdot \Omega\left(q, s_{1}, \ldots, s_{n}\right) .
$$

In terms of two-point invariants, we have

$$
\langle\vec{\mu}, \vec{\nu}\rangle^{\text {Hilb }}=\left(t_{1}+t_{2}\right)\left\langle\vec{\mu}\left|\Omega\left(q, s_{1}, \ldots, s_{n}\right)\right| \vec{\nu}\right\rangle .
$$

As a corollary, we have the following operator expressions for quantum multiplication by the divisors $D$ and $\left(1, \omega_{i}\right)$ and let

$$
M_{D}, M_{\left(1, \omega_{1}\right)}, \ldots, M_{\left(1, \omega_{n}\right)}
$$

denote the operators on $\bigoplus_{m} Q H_{T}^{*}\left(\operatorname{Hilb}_{m}\left(\mathcal{A}_{n}\right)\right)$ and let $M_{D}^{c l}, M_{\left(1, \omega_{i}\right)}^{c l}$ denote the operators for classical multiplication. 
Corollary 2.2. We have the equality

$$
\begin{gathered}
M_{D}=M_{D}^{c l}+\left(t_{1}+t_{2}\right) q \frac{d}{d q} \Omega\left(q, s_{1}, \ldots, s_{n}\right), \\
M_{\left(1, \omega_{i}\right)}=M_{\left(1, \omega_{i}\right)}^{c l}+\left(t_{1}+t_{2}\right) s_{i} \frac{d}{d s_{i}} \Omega_{+}\left(q, s_{1}, \ldots, s_{n}\right) .
\end{gathered}
$$

Proof. This follows immediately from the divisor equation

$$
\langle\vec{\mu}, D, \vec{\nu}\rangle_{\beta}=(D \cdot \beta)\langle\vec{\mu}, \vec{\nu}\rangle_{\beta}
$$

for $\beta \neq 0$.

2.7. Gromov-Witten theory of $\mathcal{A}_{n} \times \mathbf{P}^{1}$. Consider the projective line $\mathbf{P}^{1}$ with three distinguished marked points $0,1, \infty$. The $T$-equivariant Gromov-Witten theory of $\mathcal{A}_{n} \times \mathbf{P}^{1}$ relative to the fibers over $0,1, \infty$ has been studied in [M]. If we study curve classes of degree $m>0$ over the base $\mathbf{P}^{1}$, then relative conditions at each fiber are given by cohomology-weighted partitions of $m$,

$$
\vec{\mu}, \vec{\nu}, \vec{\rho} \text {. }
$$

The relative Gromov-Witten theory of $\mathcal{A}_{n} \times \mathbf{P}^{1}$ is encoded by the generating function

$$
\mathrm{Z}_{G W}^{\prime}\left(\mathcal{A}_{n} \times \mathbf{P}^{1}\right)_{\vec{\mu}, \vec{\nu}, \vec{\rho}} \in \mathbb{Q}\left(t_{1}, t_{2}\right)((u))\left[\left[s_{1}, \ldots, s_{n}\right]\right] .
$$

The reader should see [M] for an explanation of this generating function; the $u$ variable encodes the genus of the domain curve, while the variables $s_{i}$ again encode the degree of the curve classes with respect to the divisors $\omega_{i}$ on the $\mathcal{A}_{n}$ surface.

By comparing our formulas with those of $[\mathrm{M}]$, we will prove the following precise version of the Gromov-Witten/Hilbert correspondence discussed in the Introduction.

Theorem 2.2. Under the variable substitution $q=-e^{i u}$ we have

$$
\begin{aligned}
& (-1)^{m}\langle\vec{\mu}, \vec{\nu}, \vec{\rho}\rangle_{\mathcal{A}_{n}}^{\mathrm{Hilb}}=(-i u)^{-m+l(\mu)+l(\nu)+l(\rho)} \mathbf{Z}^{\prime}\left(\mathcal{A}_{n} \times \mathbf{P}^{1}\right)_{\vec{\mu}, \vec{\nu}, \vec{\rho}} \\
\text { for } \vec{\nu}= & (2) \text { and } \vec{\nu}=\left(1, \omega_{i}\right) .
\end{aligned}
$$

Moreover, the statements and proofs in $[\mathrm{M}$ ] were motivation for the strategy pursued in this paper. Again assuming a nondegeneracy conjecture, the above statement should hold for arbitrary three-point invariants.

Just as with quantum cohomology, the three-point invariants defined using the Gromov-Witten theory of $\mathcal{A}_{n} \times \mathbf{P}^{1}$ can also be used to define a ring deformation of the classical equivariant cohomology $H_{T}^{*}\left(\mathcal{A}_{n}, \mathbb{Q}\right)$ over $\mathbb{Q}\left(t_{1}, t_{2}\right)((u))\left[\left[s_{1}, \ldots, s_{n}\right]\right]$. An equivalent version of this correspondence is the statement that, after a transcendental change of variables, this ring deformation is explicitly isomorphic to the quantum deformation.

In upcoming work $\mathrm{MO}$ we will finish the comparison of both of these three-point invariants with the Donaldson-Thomas theory of $\mathcal{A}_{n} \times \mathbf{P}^{1}$.

2.8. Overview of proof. Our operator formulas have many striking qualitative features which are geometrically surprising and turn out to be useful in their proof. First, as a function of $s_{1}, \ldots, s_{n}$, we observe that (11) is essentially root-theoretic. That is, the only curve classes from $\mathcal{A}_{n}$ that contribute to $\Omega$ are those corresponding to positive roots $\alpha_{i j}$ and their multiples. Moreover, the associated expression is essentially independent of the root with which we are working. 
Second, if we fix a root $\alpha_{i, j}$ and isolate the contribution to $\Omega$ from its multiples, the associated terms have a logarithmic dependence on $\left(s_{i} \ldots s_{j-1}\right)$. In practice, this means that the contribution from $d \alpha_{i, j}$ is essentially given by the contribution from $\alpha_{i, j}$. In terms of the divisor operators from Corollary 2.2. this implies that their matrix entries are rational functions in $q, s_{1}, \ldots, s_{n}$. While one expects rational functions in $q$ for theoretical reasons, the fact that these operators are rational functions in all variables is a special feature of these geometries.

Our proof proceeds by establishing these qualitative features directly and using them to algorithmically reduce the computation to a few basic cases. We then show that both $\Theta$ and $\Omega$ satisfy the same qualitative properties and have the same value on the basic cases. In Section 3, we establish some preliminary lemmas involving reduced virtual classes. In Section 4, we set up the algorithm on the geometric side for the operator $\Theta$. In Section 5, we perform the same analysis for $\Omega$, and we prove the main theorem and its corollaries in Section 6. In particular, we discuss a conjecture on $\Omega$ which implies that these divisors generate the full quantum cohomology ring. Finally, in Section 7, we discuss elementary properties of the quantum differential equation and give an argument, due to Jim Bryan, for extending these formulas to $D$ and $E$ surface resolutions.

\section{Preliminary lemmas}

In this section, we set some further notation and explain basic properties of the reduced virtual class for $\operatorname{Hilb}\left(\mathcal{A}_{n}\right)$.

3.1. Definitions. We first clarify the definition of two-point Gromov-Witten invariants

$$
\langle\vec{\mu}, \vec{\nu}\rangle_{\beta}^{\mathrm{Hilb}}=\int_{\left[\bar{M}_{0,2}\left(\operatorname{Hilb}\left(\mathcal{A}_{n}\right), \beta\right]^{\mathrm{vir}}\right.} \mathrm{ev}_{1}^{*} \vec{\mu} \mathrm{ev}_{2}^{*} \vec{\nu} .
$$

The integrand is the virtual fundamental class on the space of stable maps and has dimension

$$
-K_{\mathrm{Hilb}\left(\mathcal{A}_{n}\right)} \cdot \beta+(2 n-3)+2=2 n-1 .
$$

As mentioned earlier, the space of stable maps is typically noncompact. There are two approaches in making sense of the above expression. The first approach, already described, is to use the fact that its $T$-fixed locus is compact. The equivariant residue is defined to be the formal contribution of these fixed loci to virtual localization. We can take the integral to be the pushforward to a point of these equivariant residues, which will take values in $\mathbb{Q}\left[t_{1}, t_{2}\right]_{\left(t_{1}+t_{2}\right)}$ due to the denominators occuring in the residue expressions.

Alternately, if the insertions correspond to $T$-equivariant cycles for which the space of maps meeting these cycles is compact, then this integral can be replaced with one over this incidence locus. We then have a $T$-equivariant integral on a compact space which is defined in the usual sense. In particular, the associated invariant takes values in $\mathbb{Q}\left[t_{1}, t_{2}\right]$. Since any insertion can be written as a combination of these compact cycles with coefficients in $\mathbb{Q}\left(t_{1}, t_{2}\right)$, we can use this as a definition in general. This agrees with the first approach by the virtual localization formula.

The Hilbert-Chow morphism defines a map from the Hilbert scheme of points to the symmetric product of $\mathcal{A}_{n}$ :

$$
\rho^{H C}: \operatorname{Hilb}_{m}\left(\mathcal{A}_{n}\right) \rightarrow S^{m}\left(\mathcal{A}_{n}\right) .
$$


We distinguish curve classes $\beta \in H_{2}\left(\operatorname{Hilb}\left(\mathcal{A}_{n}\right), \mathbb{Z}\right)$ based on whether they are contracted by $\rho_{*}^{H C}$. Curves that are contracted by $\rho^{H C}$ will be called punctual curves, since they parametrize subschemes with fixed support. Noncontracted curves will be called nonpunctual curves. It is easy to see that a class $\beta$ is punctual if and only if $\left(1, \omega_{i}\right) \cdot \beta=0$ for all $i$ and is effective if, in addition, $D \cdot \beta \geq 0$.

As we shall explain later, the contribution to the two-point operator $\Theta$ from punctual curve classes can be deduced from the calculations for $\operatorname{Hilb}\left(\mathbb{C}^{2}\right)$ in OP1. For most of this paper, we will study the contribution to $\Theta$ from non-punctual curves, denoted by

$$
\left\langle\vec{\mu}\left|\Theta_{+}\left(q, s_{1}, \ldots, s_{n}\right)\right| \vec{\nu}\right\rangle \in \mathbb{Q}\left(t_{1}, t_{2}\right)((q))\left[\left[s_{1}, \ldots, s_{n}\right]\right] .
$$

Moreover, given a curve class $\alpha=c_{1} E_{1}+\cdots+c_{n} E_{n} \in H_{2}\left(\mathcal{A}_{n}\right)$, we isolate the coefficient in $\Theta$ of $s_{1}^{c_{1}} \cdot \ldots \cdot s_{n}^{c_{n}}$ as $\Theta_{\alpha}(q)$ so that the corresponding invariants are given by

$$
\left\langle\vec{\mu}\left|\Theta_{\alpha}(q)\right| \vec{\nu}\right\rangle \in \mathbb{Q}\left(t_{1}, t_{2}\right)((q)) .
$$

Notice that we are still considering all possible values of $D \cdot \beta$, so the result is a Laurent series in $q$.

Finally, given a curve class $\beta \in H_{2}\left(\operatorname{Hilb}_{m}\left(\mathcal{A}_{n}\right), \mathbb{Z}\right)$, we define the support of $\beta$ to be the smallest interval $1 \leq i<j \leq n$ containing the set $\left\{k \mid\left(1, \omega_{k}\right) \cdot \beta \neq 0\right\}$. It will be useful to consider the contribution to $\Theta$ of all curve classes with fixed support $[i, j]$ :

$$
\left\langle\vec{\mu}\left|\Theta_{[i, j]}\right| \vec{\nu}\right\rangle \in \mathbb{Q}\left(t_{1}, t_{2}\right)((q))\left[\left[s_{i}, \ldots, s_{j-1}\right]\right] .
$$

3.2. Bases for Fock space. It will be useful in our arguments to shift between different bases for the cohomology of $\operatorname{Hilb}_{m}\left(\mathcal{A}_{n}\right)$. First, as discussed in Section 2.3. for every choice of basis $\left\{\gamma_{k}\right\}$ of $H_{T}^{*}\left(\mathcal{A}_{n}, \mathbb{Q}\right)$ there is an associated Nakajima basis $\{\vec{\mu}\}$ given by partitions weighed by elements of this basis. We will work with the Nakajima basis indexed by weighted partitions of the form

$$
\mu_{1}\left(\omega_{1}\right) \ldots \mu_{n}\left(\omega_{n}\right) \mu_{n+1}(1),
$$

where $\left\{\mu_{i}\right\}$ is an $(n+1)$-tuple of partitions (or multipartition) such that $\sum\left|\mu_{i}\right|=m$.

If we extend coefficients to $\mathbb{Q}\left(t_{1}, t_{2}\right)$, then we have a basis of $H_{T}^{*}\left(\mathcal{A}_{n}, \mathbb{Q}\right) \otimes \mathbb{Q}\left(t_{1}, t_{2}\right)$ given by fixed points $\left[p_{1}\right], \ldots,\left[p_{n+1}\right]$ and can also study its associated Nakajima basis

$$
\mu_{1}\left(\left[p_{1}\right]\right) \ldots \mu_{n+1}\left(\left[p_{n+1}\right]\right),
$$

where $\left\{\mu_{i}\right\}$ is a again a multipartition of $m$.

Finally, we will also work with the basis of localized equivariant cohomology given by $T$-fixed points on $\operatorname{Hilb}_{m}\left(\mathcal{A}_{n}\right)$, which can be described as follows. We first recall the description of $T$-fixed subschemes of $\mathbb{C}^{2}$ under the standard torus action. Given such a subscheme of length $m$, it must be the zero locus of a monomial ideal

$$
I_{\lambda}=\left(x^{\lambda_{1}}, y x^{\lambda_{2}}, \ldots, y^{l-1} x^{\lambda_{l}}\right)
$$

associated to the partition $\lambda$ of $m$.

The toric surface $\mathcal{A}_{n}$ admits an affine cover by open sets $U_{k} \cong \mathbb{C}^{2}$ centered at the fixed point $p_{k}$, where we fix the identification with $\mathbb{C}^{2}$ so that the $x$ - and $y$-axes correspond to $E_{k-1}$ and $E_{k}$, respectively. Given a $T$-fixed subscheme of length $m$ on $\mathcal{A}_{n}$, its restriction to each $U_{k}$ yields an associated monomial ideal and partition. This gives a bijection between $T$-fixed subschemes of length $m$ and multipartitions 
$\vec{\lambda}=\left\{\lambda_{k}\right\}$ such that $\sum\left|\lambda_{k}\right|=m$. The associated cohomology class shall be denoted by

$$
\left[J_{\vec{\lambda}}\right] \in H_{T}^{*}\left(\operatorname{Hilb}_{m}\left(\mathcal{A}_{n}\right)\right) \otimes \mathbb{Q}\left(t_{1}, t_{2}\right) .
$$

The relationship between the Nakajima basis associated to $\left\{p_{i}\right\}$ and the fixedpoint basis can be described in terms of symmetric functions as follows (see N2, LQW, OP1]). Both of these bases are induced by the Nakajima and fixed-point bases for Hilb $\left(\mathbb{C}^{2}\right)$ under the isomorphism

$$
\mathcal{F}_{\mathcal{A}_{n}} \otimes \mathbb{Q}\left(t_{1}, t_{2}\right)=\bigotimes_{i=1}^{n+1} \mathcal{F}_{\mathbb{C}^{2}, i} \otimes \mathbb{Q}\left(w_{L}^{i}, w_{R}^{i}\right),
$$

where the coordinate axes on the $i$-th factor have been identified with the tangent weights at $p_{i}$. Let Symm denote the ring of symmetric functions over $\mathbb{Q}\left(t_{1}, t_{2}\right)$ in countably many variables $z_{1}, z_{2}, \ldots$. This ring admits an isomorphism with Fock space by identifying 1 with the vacuum vector $v_{\emptyset}$ and the Heisenberg operator $\mathfrak{p}_{-k}\left(\left[p_{i}\right]\right)$ with multiplication by the Newton symmetric polynomial $w_{R}^{i} \cdot \mathfrak{p}_{k}(z)$ where $\mathrm{p}_{k}(z)=\sum_{a} z_{a}^{k} \in$ Symm. Under this identification, the Nakajima basis element $\mu_{i}\left(\left[p_{i}\right]\right)$ is idenitifed with the Newton symmetric function $\frac{\left(w_{R}^{i}\right)^{l\left(\mu_{i}\right)}}{\mathfrak{z}\left(\mu_{i}\right)} \mathrm{p}_{\mu_{i}}(z)$ and the normalized fixed-point $\left(w_{R}^{i}\right)^{-|\lambda|}\left[J_{\lambda}\right]$ is identified with the integral Jack polynomial

$$
\mathrm{J}_{\lambda}^{\theta}(z)
$$

with parameter $\theta=w_{L}^{i} / w_{R}^{i}$. If we specialize our equivariant weights so that $t_{1}+t_{2}=$ 0 , then the Jack polynomials written above become proportional to the associated Schur polynomials

$$
\mathbf{s}_{\lambda}(z)=(-1)^{|\lambda|} \frac{\operatorname{dim} \lambda}{|\lambda| !} \mathrm{J}_{\lambda}(z) \bmod \left(t_{1}+t_{2}\right),
$$

where $\operatorname{dim} \lambda$ is the dimension of the irreducible representation of $S_{m}$ associated to $\lambda$.

To apply this to $\mathcal{A}_{n}$, the Nakajima basis element $\prod \mu_{i}\left(\left[p_{i}\right]\right)$ now corresponds to an $(n+1)$-tuple of power-sum symmetric functions $\bigotimes_{i} \mathrm{p}_{\mu_{i}}\left(z^{(i)}\right)$ and the fixed-point basis element $\left[J_{\vec{\lambda}}\right]$ corresponds to an $(n+1)$-tuple of Jack symmetric polynomials $\otimes J_{\lambda_{i}}\left(z^{(i)}\right)$. Under the specialization $t_{1}+t_{2}=0$, we again are allowed to work with $(n+1)$-tuples of Schur polynomials.

3.3. Reduced virtual classes. We now define the reduced virtual fundamental class for $\bar{M}_{0, k}\left(\operatorname{Hilb}\left(\mathcal{A}_{n}\right), \beta\right)$. Given any variety with an everywhere-nondegenerate holomorphic symplectic form, this form gives rise to a trivial factor of the obstruction theory. By removing this trivial factor by hand, we obtain a new obstruction theory with virtual dimension increased by 1 . In the case of Hilb $\left(\mathbb{C}^{2}\right)$, this construction is important in the analysis of [OP1, and we will use it in much the same way. Our discussion is based on the more detailed treatment given there.

We first explain the standard and modified obstruction theory for a fixed domain curve $C$. Given a fixed nodal, pointed curve $C$ of genus 0 , let $M_{C}\left(\operatorname{Hilb}\left(\mathcal{A}_{n}\right), \beta\right)$ denote the moduli space of maps from $C$ to $\operatorname{Hilb}\left(\mathcal{A}_{n}\right)$ of degree $\beta \neq 0$. The usual perfect obstruction theory for $M_{C}\left(\operatorname{Hilb}\left(\mathcal{A}_{n}\right), \beta\right)$ is defined by the natural morphism

$$
R \pi_{*}\left(\mathrm{ev}^{*} T_{\mathrm{Hilb}}\right)^{\vee} \rightarrow L_{M_{C}}
$$


where $L_{M_{C}}$ denotes the cotangent complex of $M_{C}\left(\operatorname{Hilb}\left(\mathcal{A}_{n}\right), \beta\right)$ and

$$
\begin{gathered}
\text { ev }: C \times M_{C}\left(\operatorname{Hilb}\left(\mathcal{A}_{n}\right), \beta\right) \rightarrow \operatorname{Hilb}\left(\mathcal{A}_{n}\right), \\
\pi: C \times M_{C}\left(\operatorname{Hilb}\left(\mathcal{A}_{n}\right), \beta\right) \rightarrow M_{C}\left(\operatorname{Hilb}\left(\mathcal{A}_{n}\right), \beta\right)
\end{gathered}
$$

are the evaluation and projection maps.

Letting $\gamma$ denote the holomorphic symplectic form on $\mathcal{A}_{n}$ induced by the standard form $d x \wedge d y$ on $\mathbb{C}^{2}$ induces a holomorphic symplectic form on $\mathcal{A}_{n}$ which, in turn, induces a holomorphic symplectic form $\gamma$ on $\operatorname{Hilb}_{m}\left(\mathcal{A}_{n}\right)$. The $T$-representation $\mathbb{C} \cdot \gamma$ has weight $-\left(t_{1}+t_{2}\right)$. Let $\omega_{\pi}$ denote the relative dualizing sheaf. The symplectic pairing and pullback of differentials induces a map

$$
\operatorname{ev}^{*}\left(T_{\mathrm{Hilb}\left(\mathcal{A}_{n}\right)}\right) \rightarrow \omega_{\pi} \otimes(\mathbb{C} \gamma)^{*} .
$$

This, in turn, yields a map of complexes

$$
R \pi_{*}\left(\omega_{\pi}\right)^{\vee} \otimes \mathbb{C} \gamma \rightarrow R \pi_{*}\left(\operatorname{ev}^{*}\left(T_{\operatorname{Hilb}\left(\mathcal{A}_{n}\right)}\right)^{\vee}\right)
$$

and the truncation

$$
\iota: \tau_{\leq-1} R \pi_{*}\left(\omega_{\pi}\right)^{\vee} \otimes \mathbb{C} \gamma \rightarrow R \pi_{*}\left(\operatorname{ev}^{*}\left(T_{\mathrm{Hilb}\left(\mathcal{A}_{n}\right)}\right)^{\vee}\right) .
$$

This truncation is a trivial line bundle with equivariant weight $-\left(t_{1}+t_{2}\right)$.

Results of Ran and Manetti ( $\underline{\mathrm{R}}, \mathrm{Man})$ on obstruction theory and the semiregularity map imply the following. First, there is an induced map

$$
C(\iota) \rightarrow L_{M_{C}}
$$

where $C(\iota)$ is the mapping cone associated to $\iota$. Second, this map (3) satisfies the necessary properties of a perfect obstruction theory. This is precisely the modified obstruction theory we use to define the reduced virtual class. Since all maps in this section are compatible with the $T$-action, we have a $T$-equivariant reduced virtual class.

There is one important subtlety regarding the semiregularity results of $\mathrm{R}, \mathrm{Man}$. In order to apply their results, we require a compact target space. We can embed the $\mathcal{A}_{n}$ singularity in a surface $S$ with a holomorphic symplectic form that is degenerate away from the singularity. Given a stable map $f$, its deformation theory can be studied on the Hilbert scheme of the resolved surface $\widetilde{S}$ where our curve maps entirely to the nondegenerate locus. Theorem 9.1 of $\mathrm{Man}$ still gives the necessary vanishing statement for realized obstructions.

As with the standard obstruction theory (2), we obtain the reduced $T$-equivariant perfect obstruction theory on $\bar{M}_{0, k}\left(\operatorname{Hilb}\left(\mathcal{A}_{n}\right), \beta\right)$ by varying the domain $C$ and studying the the relative obstruction theory over the Artin stack $\mathfrak{M}$ of all nodal curves. Since the new obstruction theory differs from the standard one by the 1dimensional obstruction space $(\mathbb{C} \gamma)^{\vee}$, we have that the reduced virtual dimension is given by

$$
1+(2 n-3)+k .
$$

Furthermore we have the identity

$$
\begin{aligned}
{\left[\bar{M}_{0, k}\left(\operatorname{Hilb}\left(\mathcal{A}_{n}\right), \beta\right)\right]_{\text {standard }}^{\text {vir }} } & =c_{1}\left(\mathbb{C} \gamma^{\vee}\right)\left[\bar{M}_{0, k}\left(\operatorname{Hilb}\left(\mathcal{A}_{n}\right), \beta\right)\right]^{\mathrm{red}} \\
& =\left(t_{1}+t_{2}\right)\left[\bar{M}_{0, k}\left(\operatorname{Hilb}\left(\mathcal{A}_{n}\right), \beta\right)\right]^{\mathrm{red}} .
\end{aligned}
$$

We have the following lemma, whose proof is nearly identical to that of Lemma 2 in OP1]. 
Lemma 3.1. The standard T-equivariant Gromov-Witten invariants of $\operatorname{Hilb}_{m}\left(\mathcal{A}_{n}\right)$ with nonzero degree and with insertions from $H_{T}^{*}\left(\operatorname{Hilb}_{m}\left(\mathcal{A}_{n}\right), \mathbb{Q}\right)$ are divisible by $\left(t_{1}+t_{2}\right)$.

Proof. As these invariants take values in $\mathbb{Q}\left(t_{1}, t_{2}\right)$, divisibility is defined by valuation with respect to $\left(t_{1}+t_{2}\right)$. The cohomology $H_{T}^{*}\left(\operatorname{Hilb}\left(\mathcal{A}_{n}\right), \mathbb{Q}\right)$ is spanned as a $\mathbb{Q}\left[t_{1}, t_{2}\right]$-module by the Nakajima basis with respect to $\left(1, \omega_{1}, \ldots, \omega_{n}\right)$, so it suffices to prove this statement with insertions from this basis. If we instead study insertions given by the Nakajima basis with respect to the fixed points $\left[p_{1}\right], \ldots,\left[p_{n+1}\right]$, then by compactness these invariants lie in $\mathbb{Q}\left[t_{1}, t_{2}\right]$ and by the reduced construction are divisible by $\left(t_{1}+t_{2}\right)$. Since the change of basis from $\left\{\left[p_{1}\right], \ldots,\left[p_{n+1}\right]\right\}$ to $\left\{1, \omega_{1}, \ldots, \omega_{n}\right\}$ does not introduce any denominators of $\left(t_{1}+t_{2}\right)$, we are done.

We denote the reduced invariants with curved brackets; we have shown that

$$
\langle\vec{\mu}, \vec{\nu}\rangle_{0,2, \beta}^{\mathrm{Hilb}}=\left(t_{1}+t_{2}\right)(\vec{\mu}, \vec{\nu})_{0,2, \beta}^{\mathrm{Hilb}} \text {. }
$$

3.4. Factorization. The first application of reduced class arguments will be the study of two point invariants in the Nakajima basis with respect to $\left\{1, \omega_{1}, \ldots, \omega_{n}\right\}$. The following result shows that we can remove any parts labelled with 1 . It is the analog of the additivity statements for $\operatorname{Hilb}\left(\mathbb{C}^{2}\right)$ proved in Section 3.5 of [OP1].

Proposition 3.2. We have the factorization

$$
\left\langle\mu(1) \prod \lambda_{i}\left(\omega_{i}\right)\left|\Theta_{+}\right| \nu(1) \prod \rho_{i}\left(\omega_{i}\right)\right\rangle=\langle\mu(1) \mid \nu(1)\rangle \cdot\left\langle\prod \lambda_{i}\left(\omega_{i}\right)\left|\Theta_{+}\right| \prod \rho_{i}\left(\omega_{i}\right)\right\rangle .
$$

Proof. In the above expression the first factor on the right-hand side is just the usual inner product on Fock space, equivalent to the classical Poincaré pairing. In particular, it is nonzero if and only if $\mu=\nu$.

We can assume $l(\mu) \leq l(\nu)$. We consider the associated two-point invariant obtained by ordering the parts $\mu^{(k)}$ of $\mu$ and labelling them with fixed points $\left[p_{i_{k}}\right]$ :

$$
\left\langle\mu^{(1)}\left(\left[p_{i_{1}}\right]\right) \ldots \mu^{(l)}\left(\left[p_{i_{l}}\right]\right) \cdot \prod \lambda_{i}\left(\omega_{i}\right)\left|\Theta_{+}\right| \nu(1) \prod \rho_{i}\left(\omega_{i}\right)\right\rangle .
$$

The (halved) cohomological degree of this invariant is

$$
2 l(\mu)+m-l(\mu)+m-l(\nu)-(2 m-1)=l(\mu)-l(\nu)+1 \leq 1 .
$$

Since the first insertion has compact support, concentrated along the exceptional locus of $\mathcal{A}_{n}$, it forces the invariant to be a polynomial in $t_{1}$ and $t_{2}$ that must also be divisible by $\left(t_{1}+t_{2}\right)$ by Lemma 3.1. Therefore the invariant vanishes unless $l(\mu)=l(\nu)$, in which case it equals $\gamma \cdot\left(t_{1}+t_{2}\right)$, where

$$
\gamma=\left(\mu^{(1)}\left(\left[p_{i_{1}}\right]\right) \ldots \mu^{(l)}\left(\left[p_{i_{l}}\right]\right) \cdot \prod \lambda_{i}\left(\omega_{i}\right), \nu(1) \prod \rho_{i}\left(\omega_{i}\right)\right)^{\text {Hilb }} \in \mathbb{Q}
$$

is the reduced invariant. Since $\gamma$ is a nonequivariant constant, it can be evaluated by replacing the equivariant classes $\left[p_{i_{k}}\right]$ with nonequivariant point classes $\left[\xi_{k}\right]$ for distinct points $x_{i_{k}} \in \mathcal{A}_{n}$ that do not lie on any of the exceptional divisors $E_{i}$.

The moduli space of maps connecting the Nakajima cycle $\mu(\xi) \prod \lambda_{i}\left(\omega_{i}\right)$ to the Nakajima cycle $\nu(1) \prod \rho_{i}\left(\omega_{i}\right)$ is empty unless $\mu=\nu$. In this case, it can be identified with the space of maps to the product

$$
\prod_{k} \operatorname{Hilb}_{\mu_{k}}\left(\xi_{k}\right) \times Z
$$


where $\operatorname{Hilb}_{\mu_{k}}\left(\xi_{k}\right)$ is the punctual Hilbert scheme of $\mu_{k}$ points supported at the point $\xi_{k}$ and $Z$ is the subscheme of $\operatorname{Hilb}\left(\mathcal{A}_{n}\right)$ consisting of subschemes supported on the exceptional locus. This moduli space of maps to (15) has connected components corresponding to the contribution of each of these factors to the total degree of the stable map.

The only component that contributes to our invariant is where the map to each of the punctual Hilbert schemes is contracted. Indeed, the standard obstruction theory factors into contributions arising from each of the factors. Moreover, if the component of our curve class from any of these factors is nonzero, then the arguments from the last section give rise to a trivial factor of the obstruction theory. If two of these components are nonzero, then we have two trivial factors and the associated reduced invariant must also vanish. Since we are considering nonpunctual curve classes, we know that the component of our curve class in $Z$ must be nonzero, and therefore the domain curve is contracted in the projection to each factor Hilb $_{\mu_{k}}\left(\xi_{k}\right)$. The reduced invariant in (4) is given by

$$
\prod_{k} \frac{1}{\mu_{k}} \cdot\left(\prod \lambda_{i}\left(\omega_{i}\right), \prod \rho_{i}\left(\omega_{i}\right)\right)^{\mathrm{Hilb}} \text {. }
$$

Finally, if we substitute

$$
1=\sum_{k} \frac{\left[p_{k}\right]}{w_{L}^{i} \cdot w_{R}^{i}}
$$

for the first insertion, the statement of the proposition follows by a direct calculation.

As a consequence of this proposition, if we proceed inductively on the number of points $m$, it suffices to determine only a certain submatrix of the full two-point matrix $\Theta_{+}$. Moreover, from this proof these invariants are always of the form $\gamma\left(t_{1}+t_{2}\right)$, where $\gamma$ is the nonequivariant reduced invariant. This allows us to make the following useful observation. In order to calculate the two-point operator $\Theta_{+}$ precisely, it is enough to calculate the above two-point invariants $\bmod \left(t_{1}+t_{2}\right)^{2}$. If we then work with any other of the bases described in Section 3.2 it also suffices to determine the two-point invariants with respect to the new basis $\bmod \left(t_{1}+t_{2}\right)^{2}$. This is true (and well defined) since the coefficients in the change of bases never have factors of $\left(t_{1}+t_{2}\right)$ in the denominator.

\section{Geometric Calculations}

In this section, we give an inductive procedure for determining two-point invariants in terms of a fixed number of new calculations for each $m$.

The strategy in this section is to apply virtual localization GP with respect to the torus action. However, while the $T$-fixed points of $\operatorname{Hilb}\left(\mathcal{A}_{n}\right)$ are isolated, the loci of $T$-fixed curves are typically positive-dimensional and quite complicated to describe concretely. Instead we proceed indirectly and use the observation from the last section to ignore loci that contribute excess multiples of $\left(t_{1}+t_{2}\right)$. This allows us to reduce the analysis to correlators for which only zero-dimensional loci of curves contribute. 
4.1. Fixed-point correlators. Our procedure for determining these invariants proceeds via three intermediate propositions about two-point invariants in the fixedpoint basis. Recall that we use the subscript $[i, j]$ to isolate the contribution of curve classes on $\mathcal{A}_{n}$ that are linear combinations of $E_{i}, \ldots, E_{j-1}$ with nonzero coefficients for $E_{i}$ and $E_{j-1}$.

Proposition 4.1. For $m \geq 1$, an arbitrary two-point correlator

$$
\left\langle\left[J_{\vec{\lambda}}\right]\left|\Theta_{[i, j]}\right|\left[J_{\vec{\pi}}\right]\right\rangle \in \mathbb{Q}\left[t_{1}, t_{2}\right]((q))\left[\left[s_{i}, \ldots, s_{j-1}\right]\right]
$$

is congruent modulo $\left(t_{1}+t_{2}\right)^{2}$ to a linear combination of

- $\left\langle\left[J_{\vec{\eta}}\right]\left|\Theta_{[i, j]}\right|\left[J_{\vec{\eta}}\right]\right\rangle$, where $\eta_{k}=\emptyset$ for $k \neq i$,

- two-point correlators $\left\langle\left[J_{\vec{\kappa}}\right]\left|\Theta_{[i, j]}\right|\left[J_{\vec{\sigma}}\right]\right\rangle$ for $\operatorname{Hilb}_{m^{\prime}}\left(\mathcal{A}_{n}\right)$ with $m^{\prime}<m$,

with coefficients in $R=\mathbb{Q}\left(t_{1}, t_{2}\right)_{\left(t_{1}+t_{2}\right)}$ determined by pairings in the classical equivariant cohomology of $\operatorname{Hilb}\left(\mathcal{A}_{n}\right)$.

For the next two propositions, we specify the following fixed points by the multipartitions $\vec{\rho}, \vec{\theta}, \vec{\kappa}, \vec{\sigma}$ :

$$
\begin{gathered}
\rho_{i}=(m), \quad \rho_{k}=\emptyset, \text { if } k \neq i, \\
\theta_{i}=\left(1^{m}\right), \quad \theta_{k}=\emptyset, \text { if } k \neq i, \\
\kappa_{i}=(m-1), \quad \kappa_{j}=(1), \quad \kappa_{k}=\emptyset, \text { if } k \neq i, j, \\
\sigma_{i}=\left(1^{m-1}\right), \quad \sigma_{j}=(1), \quad \sigma_{k}=\emptyset, \text { if } k \neq i, j .
\end{gathered}
$$

We then have the next two steps in our algorithm.

Proposition 4.2. For $m \geq 1$, a two-point correlator $\left\langle\left[J_{\vec{\eta}}\right]\left|\Theta_{[i, j]}\right|\left[J_{\vec{\eta}}\right]\right\rangle$ where $\eta_{k}=\emptyset$ for $k \neq i$ is congruent modulo $\left(t_{1}+t_{2}\right)^{2}$ to a linear combination of

- $\left\langle\left[J_{\vec{\rho}}\right]\left|\Theta_{[i, j]}\right|\left[J_{\vec{\kappa}}\right]\right\rangle$,

- $\left\langle\left[J_{\vec{\theta}}\right]\left|\Theta_{[i, j]}\right|\left[J_{\vec{\sigma}}\right]\right\rangle$,

- two-point correlators $\left.\left\langle\left[J_{\vec{\lambda}}\right]\left|\Theta_{[i, j]}\right| J_{\vec{\eta}}\right]\right\rangle$ for $\operatorname{Hilb}_{m^{\prime}}\left(\mathcal{A}_{n}\right)$ with $m^{\prime}<m$,

with coefficients in $R$ determined by pairings in the classical equivariant cohomology of $\operatorname{Hilb}\left(\mathcal{A}_{n}\right)$.

Proposition 4.3. For these special two-point correlators we have the following expression modulo $\left(t_{1}+t_{2}\right)^{2}$ :

$$
\begin{gathered}
\left\langle\left[J_{\vec{\rho}}\right]\left|\Theta_{[i, j]}\right|\left[J_{\vec{\kappa}}\right]\right\rangle=(-1)^{m-1}\left(t_{1}+t_{2}\right)\left((n+1) t_{1}\right)^{2 m} \frac{(m !)^{2}}{m} \log \left(1-(-q)^{m-1} s_{i j}\right), \\
\left\langle\left[J_{\vec{\theta}}\right]\left|\Theta_{[i, j]}\right|\left[J_{\vec{\sigma}}\right]\right\rangle=(-1)^{m-1}\left(t_{1}+t_{2}\right)\left((n+1) t_{1}\right)^{2 m} \frac{(m !)^{2}}{m} \log \left(1-(-q)^{-m+1} s_{i j}\right),
\end{gathered}
$$

where $s_{i j}=s_{i}, \cdot \ldots \cdot s_{j-1}$ and the logarithm is expanded with nonnegative exponents in $s_{k}$.

4.2. Degree scaling. As a first application of the above three steps, we easily show the following proposition.

Proposition 4.4. The two-point operator $\Theta$ satisfies the following properties:

- $\Theta_{+}\left(q, s_{1}, \ldots, s_{n}\right)=\sum_{1 \leq i<j \leq n} \sum_{d \geq 1} \Theta_{d \alpha_{i, j}}(q)\left(s_{i} \cdot \ldots \cdot s_{j-1}\right)^{d}$,

- $\Theta_{d \alpha_{i, j}}(-q)=\Theta_{\alpha_{i, j}}\left((-q)^{d}\right) / \bar{d}$ for $\bar{d} \geq 1$. 
The first part of this proposition states that our answer only depends on a sum over roots of the $A_{n}$ lattice. The second part states that taking multiples of a fixed root has an extremely simple scaling property. We will explain a second argument for the root dependence at the end of the paper.

Proof. We proceed by induction on $m$, the number of points, starting from the vacuous case of $m=0$. Propositions 4.1, 4.2, and 4.3 then show that two-point invariants in the fixed-point basis satisfy both claims in the proposition modulo $\left(t_{1}+\right.$ $\left.t_{2}\right)^{2}$. This implies that the reduced two-point invariants $\left\langle\prod \mu_{i}\left(\omega_{i}\right), \prod \nu_{j}\left(\omega_{j}\right)\right\rangle^{\text {Hilb }}$ also satisfy both claims modulo $\left(t_{1}+t_{2}\right)^{2}$ and therefore precisely as well by the observation at the end of last section. The claim then follows from the factorization of Proposition 3.2

4.3. Localization and unbroken curves. It suffices to evaluate the reduced twopoint invariants

$$
\left(\left[J_{\vec{\lambda}}\right],\left[J_{\vec{\pi}}\right]\right)_{[i, j]}^{\text {Hilb }} \bmod \left(t_{1}+t_{2}\right),
$$

where again the subscript $[i, j]$ isolates curve classes with support in $[i, j]$. While the $T$-fixed loci are quite complicated to describe, many of these loci will contribute additional factors of $\left(t_{1}+t_{2}\right)$ and can be ignored. A description of which loci contribute nontrivially as well as the proper framework for handling them is given in OP1. The discussion given here follows Section 3.8 of that paper.

Let $T^{ \pm}$denote the antidiagonal torus $\left\{\left(\xi, \xi^{-1}\right)\right\} \subset T$. We will first analyze fixed loci with respect to the smaller torus $T^{ \pm}$. The fixed points on $\operatorname{Hilb}_{m}\left(\mathcal{A}_{n}\right)$ for $T^{ \pm}$ are the same as those with respect to $T$, but the locus of fixed maps is larger. Let $f \in \bar{M}_{0,2}\left(\operatorname{Hilb}_{m}\left(\mathcal{A}_{n}\right), \beta\right)$ denote a $T^{ \pm}$-fixed map $f: C \rightarrow \operatorname{Hilb}\left(\mathcal{A}_{n}\right)$. We say that $f$ is broken if either

- the domain $C$ contains a connected, $f$-contracted subcurve $C^{\prime}$ for which the curve $C \backslash C^{\prime}$ has at least two connected components which are not $f$ contracted or

- two non- $f$-contracted components $P_{1}, P_{2} \subset C$ meet at a node $s$ of $C$ and have tangent weights $w_{P_{1}, s}, w_{P_{2}, s}$ satisfying $w_{P_{1}, s}+w_{P_{2}, s} \neq 0$.

If $f$ is broken, then so is every map in the connected component of the $T^{ \pm}$-fixed locus containing $f$, and we can classify these components as broken and unbroken. We can decompose the $T^{ \pm}$-equivariant calculation for the reduced two-point correlator into contributions from broken and unbroken fixed loci:

$$
\left(\left[J_{\vec{\lambda}}\right],\left[J_{\vec{\pi}}\right]\right)_{[i, j]}^{\text {Hilb }}=\left(\left[J_{\vec{\lambda}}\right],\left[J_{\vec{\pi}}\right]\right)_{[i, j]}^{\text {broken }}+\left(\left[J_{\vec{\lambda}}\right],\left[J_{\vec{\pi}}\right]\right)_{[i, j]}^{\text {unbroken }} .
$$

The following lemma is proven in OP1].

Lemma 4.5. The $T^{ \pm}$-equivariant broken contributions vanish. In particular, if there are no unbroken contributions, then $\left(\left[J_{\vec{\lambda}}\right],\left[J_{\vec{\pi}}\right]\right)_{[i, j]}^{\text {Hilb }}$ vanishes $\bmod t_{1}+t_{2}$.

4.4. Properties of unbroken curves. Every nonempty unbroken connected component must contain a $T$-fixed map, so we can determine necessary criteria for two-point invariants to be nonzero $\bmod \left(t_{1}+t_{2}\right)^{2}$.

We will first give a description of nonpunctual one-dimensional $T$-orbits on $\operatorname{Hilb}_{m}\left(\mathcal{A}_{n}\right)$. In the following, given partitions $\mu, \rho$ we denote by $\mu \cup \rho$ the partition obtained by concatenating the lists of parts and $\mu^{\prime}$ the partition obtained by taking the transpose of the associated Young diagram. 
Lemma 4.6. Suppose we have a nonpunctual one-dimensional $T$-orbit $C$ connecting fixed points $J_{\vec{\lambda}}$ and $J_{\vec{\eta}}$. Then there exists a unique $k$ and partitions $\mu, \nu, \rho$ such that

- $\left(1, \omega_{k}\right) \cdot[C]>0$,

- $\lambda_{l}=\eta_{l}$ for $l \neq k, k+1$,

- up to reordering the two fixed points we have

$$
\begin{gathered}
\lambda_{k}=\mu \cup \rho, \quad \lambda_{k+1}=\nu^{\prime}, \\
\eta_{k}=\mu, \quad \eta_{k+1}=(\nu \cup \rho)^{\prime},
\end{gathered}
$$

- and the tangent weight at $J_{\vec{\lambda}}$ is a positive integral multiple of $w_{R}^{k}$.

If $l(\rho)=1$, then there is a unique such T-orbit.

Proof. The structure of a nonpunctual orbit near a fixed point can be analyzed using Hilb $\left(\mathbb{C}^{2}\right)$ as a local model, in which case the third and fourth conditions are satisfied by the analysis in Section 7.2 of [N3]. The transpose occurs because of our convention for partition orientation. Since the tangent weights $w_{R}^{k}$ are not proportional to any other tangent weights, this forces the uniqueness of $k$ as well as the first two conditions.

For punctual $T$-orbits we have the following lemma.

Lemma 4.7. Given a punctual T-orbit connecting $J_{\vec{\lambda}}$ and $J_{\vec{\eta}}$, there exists a unique $k$ such that

- $\lambda_{l}=\eta_{l}$ for $l \neq k$,

- the tangent weight at $J_{\vec{\lambda}}$ is of the form

$$
a \cdot w_{L}^{k}+b \cdot w_{R}^{k}
$$

with $a \cdot b \leq 0$.

Proof. At a fixed point of $\operatorname{Hilb}\left(\mathbb{C}^{2}\right)$, the tangent weights are of the form $a t_{1}+b t_{2}$ with $a \cdot b \leq 0$. One can directly check that the associated linear combinations $a \cdot w_{L}^{k}+b \cdot w_{R}^{k}$ are distinct as we vary $k$. Therefore any $T$-orbit is noncontracted over a unique fixed point and the statement follows.

Let $f:\left(C, z_{1}, z_{2}\right) \rightarrow \operatorname{Hilb}_{m}\left(\mathcal{A}_{n}\right)$ be a two-pointed, $T$-fixed unbroken map. Any noncontracted irreducible component of $C$ has at most 2 marked or nodal points. This implies that $C$ must be a chain of rational curves

$$
C=C_{1} \cup C_{2} \cup \cdots \cup C_{r}
$$

with nodes $q_{1}, \ldots, q_{r-1}$. Up to relabelling, we must have

$$
z_{1} \in C_{1}, z_{2} \in C_{r}
$$

or

and $C_{1}$ is $f$-contracted.

$$
z_{1}, z_{2} \in C_{1}
$$

In the first case, let $S$ denote the sequence of $T$-fixed points of $\operatorname{Hilb}\left(\mathcal{A}_{n}\right)$ :

$$
S=f\left(z_{1}\right), f\left(q_{1}\right), f\left(q_{2}\right), \ldots, f\left(q_{r}\right), f\left(z_{2}\right) .
$$

We otherwise take $S$ to be

$$
S=f\left(q_{1}\right), f\left(q_{2}\right), \ldots, f\left(q_{r}\right) .
$$


We define an ordering on fixed points given as follows. Given a partition $\lambda$ with parts $\lambda^{(1)}, \ldots, \lambda^{(l)}$, we have the function

$$
\epsilon(\lambda)=\sum\left(\begin{array}{c}
\lambda^{(i)} \\
2
\end{array}\right)
$$

Given two multipartitions $\vec{\lambda}, \vec{\eta}$, we say that

$$
\vec{\lambda} \succeq \vec{\eta}
$$

if, when $k$ is the smallest integer such that $\left|\lambda_{k}\right| \neq\left|\eta_{k}\right|$, we have $\left|\lambda_{k}\right|>\left|\eta_{k}\right|$ or, if $\left|\lambda_{k}\right|=\left|\eta_{k}\right|$ for all $k$, then $\epsilon\left(\lambda_{k}\right) \geq \epsilon\left(\eta_{k}\right)$ for all $k$.

Lemma 4.8. The sequence $S$ is either an increasing sequence or a decreasing sequence with respect to the ordering $\succeq$.

Proof. This follows from the description of the one-dimensional orbits. We discuss the case where $z_{1}$ and $z_{2}$ are on noncontracted components. Assume that the (fractional) tangent weight to $C_{1}$ at $z_{1}$ is congruent to $a t_{1} \bmod \left(t_{1}+t_{2}\right)$ with $a<0$. Because of the unbroken condition, the sum of the tangent weights at each node $q_{k}$ must be proportional to $t_{1}+t_{2}$, so the tangent weight to $C_{k}$ at $q_{k-1}$ is $a t_{1} \bmod \left(t_{1}+t_{2}\right)$ for all $k$.

For a nonpunctual component $C_{k}$, it is a multiple cover of one of the orbits described in Lemma 4.6. Since $a<0$, the tangent space at $q_{k-1}$ maps to a $T$ fixed point with tangent weight a positive rational multiple $w_{R}^{l}$ for some $l$, since $w_{L}^{l}=b t_{1} \bmod \left(t_{1}+t_{2}\right)$ with $b>0$. The length of the support of the subscheme at $p_{l}$ decreases and increases at $p_{l+1}$, so we have $f\left(q_{k-1}\right) \succeq f\left(q_{k}\right)$.

For a punctual component $C_{k}$ connecting partitions $\lambda$ and $\eta$ supported at the fixed point $p_{l}$, the tangent weight at $q_{k-1}$ is a positive rational multiple of $a w_{L}$ degree with respect to $D$, given by the localization expression $0<D \cdot C_{k}=\frac{c(\lambda)-c(\eta)}{a w_{L}^{l}+b w_{R}^{l}}$ where $c(\lambda)$ is the content

$$
c(\lambda)=\left.D\right|_{J_{\lambda}}=\sum_{(i, j) \in \lambda}(i-1) w_{L}^{l}+(j-1) w_{R}^{l} .
$$

The coefficient of $w_{L}^{l}$ in $c(\lambda)$ is precisely $\epsilon(\lambda)$, which implies $\epsilon(\lambda) \geq \epsilon(\eta)$, so again we have $f\left(q_{k-1}\right) \succeq f\left(q_{k}\right)$.

The main application of this unbroken analysis is the following vanishing proposition for two-point fixed correlators.

Proposition 4.9. Given two distinct $(n+1)$-tuples of partitions $\vec{\lambda} \neq \vec{\eta}$ such that either $\left|\lambda_{i}\right|=\left|\eta_{i}\right|$ or $\left|\lambda_{j}\right|=\left|\eta_{j}\right|$, then

$$
\left\langle\left[J_{\vec{\lambda}}\right]\left|\Theta_{[i, j]}\right|\left[J_{\vec{\eta}}\right]\right\rangle=0 \bmod \left(t_{1}+t_{2}\right)^{2} .
$$

Proof. Since we are considering curve classes $\beta$ with support equal to $[i, j]$, we have $\left(1, \omega_{k}\right) \cdot \beta=0$ for $k<i$ and $\left(1, \omega_{i}\right) \cdot \beta>0$. Suppose there exists an unbroken map $f$; since $\vec{\lambda} \neq \vec{\eta}$, the two marked points must be at opposite ends of the chain $C$. The first condition and the description of punctual and nonpunctual $T$-orbits ensures that $\left|\lambda_{k}\right|=\left|\eta_{k}\right|$ for $k<i$. Similarly, the second condition implies that the length of the subscheme supported at $p_{i}$ either increases or decreases at some point in the chain. Lemma 4.8 implies that $\left|\lambda_{i}\right| \neq\left|\eta_{i}\right|$ and the analogous statement for $j$. This contradicts the hypothesis, so all $T$-fixed maps are broken. 
4.5. Proof of Proposition 4.1. The proof of this proposition and the next will only use the factorization and vanishing statements of Propositions 3.2 and 4.9 .

Recall that our invariants take values in $R=\mathbb{Q}\left[t_{1}, t_{2}\right]_{\left(t_{1}+t_{2}\right)}$. We define an equivalence relation $\sim$ on elements of $R((q))\left[\left[s_{1}, \ldots, s_{n}\right]\right]$ so that

$$
f\left(t_{1}, t_{2}, q, s_{1}, \ldots, s_{n}\right) \sim 0
$$

if $f \bmod \left(t_{1}+t_{2}\right)^{2}$ is an $R$-linear combination of $\left\langle\left[J_{\vec{\kappa}}\right]\left|\Theta_{i, j}\right|\left[J_{\vec{\sigma}}\right]\right\rangle$ for $\operatorname{Hilb}_{m^{\prime}}\left(\mathcal{A}_{n}\right)$ with $m^{\prime}<m$.

In these terms, the factorization proposition can be restated as follows.

Lemma 4.10. Given $k>0, \vec{\mu} \in H^{*}\left(\operatorname{Hilb}_{m}\left(\mathcal{A}_{n}\right), \mathbb{Q}\right), \vec{\nu} \in H^{*}\left(\operatorname{Hilb}_{m-k}\left(\mathcal{A}_{n}\right), \mathbb{Q}\right)$, we have

$$
\left\langle\vec{\mu}\left|\Theta_{i, j}\right| \mathfrak{p}_{-k}(1) \vec{\nu}\right\rangle \sim 0
$$

Proof. If we write $\vec{\mu}, \vec{\nu}$ in terms of the Nakajima basis with respect to $1, \omega_{1}, \ldots, \omega_{n}$, this follows immediately from the statement of Proposition 3.2 since $k>0$.

In the case of $\mathcal{A}_{1}$, we will restate this lemma in terms of the symmetric function notation for elements of $\mathcal{F}_{\mathcal{A}_{1}}$. Since

$$
\mathfrak{p}_{-k}(1)=\frac{1}{2 t_{1}\left(t_{2}-t_{1}\right)} \mathfrak{p}_{-k}\left(\left[p_{1}\right]\right)+\frac{1}{2 t_{2}\left(t_{1}-t_{2}\right)} \mathfrak{p}_{-k}\left(\left[p_{2}\right]\right)
$$

and each of these fixed-point Nakajima operators corresponds to multiplication by $\mathrm{p}_{k}(z)$, we have

$$
\left\langle\left(\mathrm{p}_{\mu}(z) \cdot g(z)\right) \otimes h(z)\left|\Theta_{+}\right| \bullet\right\rangle \sim(-1)^{l(\mu)}\left\langle g(z) \otimes\left(\mathrm{p}_{\mu}(z) \cdot h(z)\right)\left|\Theta_{+}\right| \bullet\right\rangle,
$$

where $\bullet$ denotes any cohomology class.

If we apply Lemma 4.10 to invariants with insertions in the Nakajima basis with respect to the basis $\left\{1, p_{1}, \ldots, \widehat{p_{j}}, p_{n}\right\}$, this shows that all two-point correlators are determined by two-point correlators

$$
\left\langle\left[J_{\vec{\lambda}}\right]\left|\Theta_{i, j}\right|\left[J_{\vec{\eta}}\right]\right\rangle
$$

with $\lambda_{j}=\emptyset, \eta_{j}=\emptyset$. By Proposition 4.9, we must have $\vec{\lambda}=\vec{\eta}$. For $\mathcal{A}_{1}$, this concludes the proof.

For the case of general $\mathcal{A}_{n}$, we have to argue further as follows. Given two partitions $\pi$ and $\pi^{\prime}$ of size $a$ and $a-1$ respectively, we say that

$$
\pi \pi^{\prime}
$$

if their Young diagrams differ by the removal of a single box. We already have that $\lambda_{j}=\emptyset$; suppose further that there exists $k \neq i, j$ for which $\lambda_{k} \neq \emptyset$. Then there exists a multipartition $\vec{\eta}$ such that

$$
\lambda_{r}=\eta_{r}, \quad r \neq k,
$$

and

$$
\lambda_{k} \searrow \eta_{k} .
$$

If we use symmetric function notation for the fixed-point basis, then we have that modulo $\left(t_{1}+t_{2}\right)$

$$
\mathfrak{p}_{-1}(1)\left(\bigotimes_{r} \jmath_{\eta_{r}}\left(z^{(r)}\right)\right) \equiv(n+1) t_{1} \sum_{s=1}^{n+1} \bigotimes_{r \neq s} J_{\eta_{r}}\left(z^{(r)}\right) \otimes\left(\mathfrak{p}_{1}\left(z^{(s)}\right) \cdot J_{\eta_{s}}\left(z^{(s)}\right)\right) .
$$


Moreover, if we expand out these products in the basis of Jack polynomials, we have that

$$
\mathrm{p}_{1}(z) \cdot \mathrm{J}_{\eta_{k}}(z)=c \cdot \mathrm{J}_{\lambda_{k}}(z)+\ldots
$$

with $c \not \equiv 0 \bmod t_{1}+t_{2}$. This implies that

$$
\begin{aligned}
0 & \sim\left\langle\bigotimes \mathrm{J}_{\lambda_{r}}\left(z^{(r)}\right)\left|\Theta_{i, j}\right| \mathfrak{p}_{-1}(1) \cdot \bigotimes \mathrm{J}_{\eta_{r}}\left(z^{(r)}\right)\right\rangle \\
& \sim \sum_{s=1}^{n+1}\left\langle\mathrm{~J}_{\lambda_{r}}\left(z^{(r)}\right)\left|\Theta_{i, j}\right| \bigotimes_{r \neq s} \mathrm{~J}_{\eta_{r}}\left(z^{(r)}\right) \otimes\left(\mathrm{p}_{1}(z) \cdot \mathrm{J}_{\eta_{s}}(z)\right)\right\rangle \\
& \sim c\left\langle\bigotimes \mathrm{J}_{\lambda_{r}}(z)\left|\Theta_{i, j}\right| \bigotimes \mathrm{J}_{\lambda_{r}}(z)\right\rangle .
\end{aligned}
$$

The last equality follows from Proposition 4.9, since the number of points supported at either $p_{i}$ or $p_{j}$ is fixed.

4.6. Proof of Proposition 4.2. We will just prove this for $\mathcal{A}_{1}$, since the case of general $n$ is essentially the same. We will write everything using symmetric function notation for fixed-point basis elements. Moreover, since we are ignoring factors of $t_{1}+t_{2}$, it is convenient to work with Schur polynomials instead of Jack polynomials, since the branching rules are easier to describe. In these terms, the goal of this section is to calculate

$$
\left\langle\mathbf{s}_{\lambda}(z) \otimes 1\left|\Theta_{1,2}\right| \mathbf{s}_{\lambda}(z) \otimes 1\right\rangle
$$

in terms of

$$
\left\langle\mathbf{s}_{\rho}(z) \otimes 1\left|\Theta_{1,2}\right| \mathbf{s}_{\kappa}(z) \otimes \mathbf{s}_{(1)}(z)\right\rangle
$$

and

$$
\left\langle\mathrm{s}_{\theta}(z) \otimes 1\left|\Theta_{1,2}\right| \mathrm{s}_{\sigma}(z) \otimes \mathrm{s}_{(1)}(z)\right\rangle,
$$

where $\rho=(m), \kappa=(m-1), \theta=\left(1^{m}\right), \sigma=\left(1^{m-1}\right)$.

We first assume that there are two distinct partitions, $\lambda_{1}, \lambda_{2}$, of size $m-1$ such that

$$
\lambda \searrow \lambda_{1}, \lambda \searrow \lambda_{2}
$$

This happens if and only if $\lambda \neq(a, a, \ldots, a)$ for some $a$ dividing $m$.

We then have the equality

$$
\begin{aligned}
0 & \sim\left\langle\mathrm{s}_{\lambda_{1}}(z) \otimes \mathrm{s}_{(1)}(z)\left|\Theta_{1,2}\right| \mathrm{s}_{\lambda_{2}}(z) \otimes \mathrm{s}_{(1)}(z)\right\rangle \\
& \sim\left\langle\left(\mathrm{s}_{(1)}(z) \cdot \mathrm{s}_{\lambda_{1}}(z)\right) \otimes 1\left|\Theta_{1,2}\right|\left(\mathrm{s}_{(1)}(z) \cdot \mathrm{s}_{\lambda_{2}}(z)\right) \otimes 1\right\rangle \\
& \sim \sum_{\eta_{1} \searrow \lambda_{1}} \sum_{\eta_{2} \searrow \lambda_{2}}\left\langle\mathrm{~s}_{\eta_{1}}(z) \otimes 1\left|\Theta_{1,2}\right| \mathrm{s}_{\eta_{2}}(z) \otimes 1\right\rangle \\
& \sim\left\langle\mathrm{s}_{\lambda}(z) \otimes 1\left|\Theta_{1,2}\right| \mathrm{s}_{\lambda}(z) \otimes 1\right\rangle .
\end{aligned}
$$

The first equality follows from Proposition 4.9 , since $\lambda_{1} \neq \lambda_{2}$. The second equality is the restatement of the factorization lemma in terms of symmetric functions given after Lemma 4.10 The third equality is the branching rule formula for multiplying Schur functions. The fourth equality follows again from the vanishing statement and the fact that $\lambda$ is the unique partition $\eta$ of $m$ such that

$$
\eta \searrow \lambda_{1}, \quad \eta \searrow \lambda_{2}
$$


This reduces us to the case where $\lambda=(a, a, \ldots, a)$ for some $a \mid m$ or, equivalently, where the Young diagram of $\lambda$ is rectangular. If $a \neq 1, m$, there are two distinct ways of removing two boxes from the Young diagram of $\lambda$ :

$$
\lambda_{1}=(a, a, \ldots, a-1, a-1), \quad \lambda_{2}=(a, a, \ldots, a, a-2) .
$$

We then again have

$$
\begin{aligned}
0 & \sim\left\langle\mathrm{s}_{\lambda_{1}}(z) \otimes \mathrm{s}_{(2)}(z)\left|\Theta_{1,2}\right| \mathrm{s}_{\lambda_{2}}(z) \otimes \mathrm{s}_{(1,1)}(z)\right\rangle \\
& \sim\left\langle\mathrm{s}_{(1,1)}(z) \cdot \mathrm{s}_{\lambda_{1}}(z) \otimes 1\left|\Theta_{1,2}\right| \mathrm{s}_{(2)}(z) \cdot \mathrm{s}_{\lambda_{2}}(z) \otimes 1\right\rangle \\
& \sim\left\langle\mathrm{s}_{\lambda}(z) \otimes 1\left|\Theta_{1,2}\right| \mathrm{s}_{\lambda}(z) \otimes 1\right\rangle
\end{aligned}
$$

by again using Proposition 4.9 and the branching rule for Schur functions.

This leaves $\lambda=\rho$ or $\lambda=\theta$. In these cases, we have for instance

$$
\left\langle\mathrm{s}_{\theta}(z) \otimes 1\left|\Theta_{1,2}\right| \mathrm{s}_{\sigma}(z) \otimes \mathrm{s}_{1}(z)\right\rangle \sim(-1) \cdot\left\langle\mathrm{s}_{\theta}(z) \otimes 1\left|\Theta_{1,2}\right| \mathrm{s}_{\theta}(z) \otimes 1\right\rangle .
$$

We argue similarly in the case of $\lambda=\rho$.

4.7. Proof of Proposition 4.3. In order to prove Propositon 4.3, we will explicitly calculate the two invariants

$$
\left\langle\left[J_{\vec{\theta}}\right]\left|\Theta_{i, j}\right|\left[J_{\vec{\sigma}}\right]\right\rangle,\left\langle\left[J_{\vec{\rho}}\right]\left|\Theta_{i, j}\right|\left[J_{\vec{\kappa}}\right]\right\rangle \bmod \left(t_{1}+t_{2}\right)^{2} .
$$

The main observation in each case is that, for every curve class, there is at most one unbroken $T$-fixed curve joining the two fixed point insertions in each of these invariants. If we assume this for now, then since every positive-dimensional compact variety with a $T$-action has at least two fixed points, this curve is the entire unbroken $T^{ \pm}$-fixed locus. By Lemma 4.5, we have

$$
\begin{aligned}
\left(\left[J_{\vec{\theta}}\right],\left[J_{\vec{\sigma}}\right]\right)^{T} \bmod \left(t_{1}+t_{2}\right) & \equiv\left(\left[J_{\vec{\theta}}\right],\left[J_{\vec{\sigma}}\right]\right)^{T^{ \pm}} \\
& =\left(\left[J_{\vec{\theta}}\right],\left[J_{\vec{\sigma}}\right]\right)^{T^{ \pm}}, \text {unbroken },
\end{aligned}
$$

where the curved brackets denote the reduced virtual class invariants. These invariants differ from the usual invariants by a factor of $\left(t_{1}+t_{2}\right)$. Therefore, as long as we work $\bmod \left(t_{1}+t_{2}\right)^{2}$, it suffices to calculate the localization residue of the unique unbroken $T$-fixed curve.

We begin by explaining the calculation of the first invariant.

Lemma 4.11. The only unbroken maps joining $J_{\vec{\theta}}$ to $J_{\vec{\sigma}}$ have multidegree given by the monomial $\left(q^{1-m} s_{i} \cdot \ldots \cdot s_{j-1}\right)^{d}$ for $d \geq 1$. Moreover, for each $d$, there is a unique such map.

Proof. We first show that there is a unique chain of $T$-orbits that is decreasing with respect to the partial ordering $\succeq$. Let $f: C \rightarrow \operatorname{Hilb}\left(\mathcal{A}_{n}\right)$ be an unbroken $T$-fixed map with

$$
C=C_{1} \cup C_{2} \cup \cdots \cup C_{r}
$$

and let $S=\left\{J_{\theta}, f\left(q_{1}\right), \ldots, f\left(q_{r-1}\right), J_{\sigma}\right\}$ be the decreasing sequence of fixed points associated to the marked points and nodes. For $1 \leq k \leq j-i$, let $\theta^{(k)}$ be the multipartition defined by

$$
\theta_{i}^{(k)}=\left(1^{m-1}\right), \theta_{i+k}^{(k)}=(1), \theta_{l}^{(k)}=\emptyset, \quad l \neq i, i+k .
$$

We claim that

$$
S=\left\{\theta, \theta^{(1)}, \theta^{(2)}, \ldots, \theta^{(j-1)}=\sigma\right\}
$$


Indeed, let $L_{1}, \ldots, L_{r}$ denote the $T$-orbits that are the reduced images of $C_{k}$. First, $L_{1}$ must be a nonpunctual $T$-orbit with a single point moving from $p_{i}$ to $p_{i+1}$, so $f\left(q_{1}\right)=\theta^{(1)}$. This is due to the fact that the function $\epsilon$ achieves a strict minimum on $\left(1^{m}\right)$. For this same reason, all subsequent $L_{k}$ are nonpunctual curves as well which force $f\left(q_{k}\right)=\theta^{(k)}$. The uniqueness of these orbits follows from the full statement of Lemma 4.6

The degrees of each of these orbits are calculated as follows:

$$
\begin{gathered}
D \cdot L_{1}=\frac{-\left(\begin{array}{c}
m \\
2
\end{array}\right) w_{R}^{i}+\left(\begin{array}{c}
m-1 \\
2
\end{array}\right) w_{R}^{i}}{w_{R}^{i}}=1-m, \\
\left(1, \omega_{i}\right) \cdot L_{1}=1,\left(1, \omega_{l}\right) \cdot L_{1}=0 .
\end{gathered}
$$

Similarly, we have

$$
D \cdot L_{k}=0,\left(1, \omega_{k}\right) \cdot L_{k}=1
$$

Suppose that the degree of $C_{1}$ over $L_{1}$ is $d$. The tangent weights at $f\left(q_{k}\right)$ are $w_{L}^{i+k}$ and $w_{R}^{i+k}$, which add to $t_{1}+t_{2}$. Since the fractional tangent weights to $C$ at each node $q_{k}$ must add to a multiple of $t_{1}+t_{2}$, we have that the degree of $C_{k}$ over $L_{k}$ is $d$ for all $k$. Therefore, only the unbroken fixed maps have degree given by $q^{d(1-m)} \cdot\left(s_{i} \cdot \ldots \cdot s_{j-1}\right)^{d}$, and, for each $d$, there is a unique such map $f$.

This calculation of the residue at this unbroken map $f$ factors into contributions from each component $C_{k}$, each node $q_{k}$, and each marked point at $J_{\vec{\theta}}$ and $J_{\vec{\sigma}}$. For more details on how to determine these contributions, we refer the reader to $\mathrm{GP}$.

- Contribution from $C_{1}$ :

This contribution is given by the ratio of equivariant Euler classes

$$
\frac{1}{d} \cdot \frac{e\left(H^{1}\left(C_{1}, f^{*}\left(T_{\operatorname{Hilb}\left(\mathcal{A}_{n}\right)}\right)\right)\right)}{e\left(H^{0}\left(C_{1}, f^{*}\left(T_{\operatorname{Hilb}\left(\mathcal{A}_{n}\right)}\right)\right)-0\right)} .
$$

The $\frac{1}{d}$ factor arises from the automorphism of $C_{1}$ over $L_{1}$. The $H^{0}$-term has a single trivial weight 0 corresponding to reparameterization that we remove by hand in the above expression. The restriction of the tangent bundle to the orbit $L_{1}$ is given by

$$
\left.T_{\operatorname{Hilb}\left(\mathcal{A}_{n}\right)}\right|_{L_{1}}=\mathcal{O}(2) \oplus \mathcal{O}(-2) \oplus \mathcal{O}(1)^{m-1} \oplus \mathcal{O}(-1)^{m-1} .
$$

The $T$ weights over each fixed point of these bundles are given by the following table:

\begin{tabular}{c|c|c} 
& $J_{\vec{\theta}}$ & $J_{\vec{\sigma}}$ \\
\hline $\mathcal{O}(2)$ & $w_{R}^{i}$ & $-w_{R}^{i}$ \\
$\mathcal{O}(-2)$ & $w_{L}^{i}$ & $w_{L}^{i}+2 w_{R}^{i}$ \\
$\mathcal{O}(1)$ & $2 w_{R}^{i}$ & $w_{R}^{i}$ \\
$\vdots$ & $\vdots$ & $\vdots$ \\
$\mathcal{O}(1)$ & $m w_{R}^{i}$ & $(m-1) w_{R}^{i}$ \\
$\mathcal{O}(-1)$ & $w_{L}^{i}-w_{R}^{i}$ & $w_{L}^{i}$ \\
$\vdots$ & $\vdots$ & $\vdots$ \\
$\mathcal{O}(-1)$ & $w_{L}^{i}-(m-1) w_{R}^{i}$ & $w_{L}^{i}-(m-2) w_{R}^{i}$
\end{tabular}


In what follows, let $\tau \equiv(n+1) t_{1} \bmod \left(t_{1}+t_{2}\right)$. The contribution of $H^{0}\left(C_{1}, f^{*}(\mathcal{O}(2))-0\right.$ is given by

$$
\prod_{k=1}^{d}-\left(\frac{k}{d} w_{R}^{i}\right)^{2} \equiv(-1)^{d}\left(\frac{d !}{d^{d}}\right)^{2} \tau^{2 d} .
$$

The contribution of $H^{0}\left(C_{1}, f^{*}(\mathcal{O}(1))\right)$ for $a=1, \ldots, m-1$ is

$$
(-1)^{d+1} \tau^{d+1} \prod_{k=0}^{d}\left(a+\frac{k}{d}\right) \bmod \left(t_{1}+t_{2}\right) .
$$

The contribution of $H^{1}\left(C_{1}, f^{*}(\mathcal{O}(-2))\right)$ is given by

$$
\left(t_{1}+t_{2}\right) \cdot(-1)^{d-1}\left(\frac{d !}{d^{d}}\right)^{2} \tau^{2 d-2} \bmod \left(t_{1}+t_{2}\right)^{2} .
$$

The contribution of $H^{1}\left(C_{1}, f^{*}(\mathcal{O}(-1))\right)$ for $a=1, \ldots, m-1$ is

$$
\tau^{d-1} \prod_{k=1}^{d-1}\left(a+\frac{k}{d}\right) \bmod \left(t_{1}+t_{2}\right) .
$$

The total contribution is then

$$
\frac{(-1)^{m d+m+d}}{d(m-1) ! m !} \frac{1}{\tau^{2 m}} \cdot\left(t_{1}+t_{2}\right) \bmod \left(t_{1}+t_{2}\right)^{2} .
$$

- Contribution from $C_{k}, k>1$ :

The same calculation shows that the contribution here is given by

$$
\frac{(-1)^{m}}{d \cdot((m-1) !)^{2}} \cdot\left(t_{1}+t_{2}\right) \bmod \left(t_{1}+t_{2}\right)^{2} .
$$

- Contribution from nodes:

At each node $q_{k}$ we have a contribution

$$
\frac{d}{t_{1}+t_{2}}
$$

arising from the normal direction to the fixed locus arising from smoothing the node, and

$$
(-1)^{m} \tau^{2 m}((m-1) !)^{2} \bmod \left(t_{1}+t_{2}\right)
$$

from the gluing condition at the node.

- Contribution from marked points:

The contribution from each marked point is the product of the tangent weights at the fixed point

$$
(-1)^{m} \tau^{2 m}(m !)^{2},(-1)^{m} \tau^{2 m}((m-1) !)^{2} \bmod \left(t_{1}+t_{2}\right) .
$$

The total contribution yields

$$
\begin{aligned}
\left\langle\left[J_{\vec{\theta}}\right]\left|\Theta_{i, j}\right|\left[J_{\vec{\sigma}}\right]\right\rangle & =\sum_{d \geq 1} \frac{1}{d} \frac{(m !)^{2}}{m}(-1)^{m d+m+d} \tau^{2 m}\left(q^{1-m} s_{i} \cdot \ldots \cdot s_{j-1}\right)^{d} \\
& =(-1)^{m-1}\left((n+1) t_{1}\right)^{2 m} \frac{(m !)^{2}}{m} \log \left(1-(-q)^{1-m} s_{i} \cdot \ldots \cdot s_{j-1}\right) .
\end{aligned}
$$

For the calculation of

$$
\left\langle\left[J_{\vec{\rho}}\right]\left|\Theta_{i, j}\right|\left[J_{\vec{\kappa}}\right]\right\rangle,
$$


we argue in the same way, omitting the details since they are similar to the last calculation. As before, there is a unique sequence of $T$-orbits connecting the two fixed points that is decreasing with respect to the partial ordering $\succeq$. Let $\rho^{(k)}$ be the multipartitions defined by

$$
\begin{gathered}
\rho_{i}^{(1)}=(m-1,1), \rho_{l}^{(1)}=\emptyset, \quad l \neq i, \\
\rho_{i}^{(k)}=(m-1), \rho_{i+k-1}^{(k)}=(1), \rho_{l}^{(k)}=\emptyset .
\end{gathered}
$$

Then the sequence of marked points and nodes associated to any fixed, unbroken map is

$$
S=\left\{\vec{\rho}, \rho^{(1)}, \ldots, \rho^{(j-i+1)}=\vec{\kappa}\right\} .
$$

The argument for this is analogous to the argument given above, now using the fact that the two largest values of the function $\epsilon$ are achieved at the partitions

$$
(m),(m-1,1) \text {. }
$$

Again, the $T$-orbits $L_{1}, \ldots, L_{j-i+1}$ joining the fixed points in $S$ are uniquely determined. The orbit $L_{1}$ is punctual with tangent weights $\pm\left((m-1) w_{L}^{i}-w_{R}^{i}\right)$ and has degree given by the monomial $q$. The orbit $L_{2}$ has degree given by the monomial $q^{-1} s_{i}$ while the remaining $L_{k}$ are nonpunctual with tangent weights $\pm \tau \bmod \left(t_{1}+t_{2}\right)$ and degree $s_{k}, i<k<j$. Given an unbroken T-fixed map $f: C \rightarrow \operatorname{Hilb}\left(\mathcal{A}_{n}\right)$, $C=C_{1} \cup \cdots \cup C_{r}$ as before, if the degree of $C_{2}$ over $L_{2}$ is $d$, then the unbroken condition forces the degree of $C_{1}$ over $L_{1}$ to be $m d$ and the degree of $C_{k}$ over $L_{k}$ for $k \geq 2$ is $d$. Therefore there is again a unique unbroken map for every $d \geq 1$.

- Contribution for $L_{1}$ :

This punctual contribution is the main calculation of [OP1]:

$$
\left(t_{1}+t_{2}\right) \cdot(-1)^{m d+m} \frac{(m !)^{2}}{m d} \tau^{2 m} \bmod \left(t_{1}+t_{2}\right)^{2} .
$$

- Contribution for the node $q_{1}$ :

The contribution from smoothing the node at this point yields

$$
\frac{m d}{(m-1)\left(t_{1}+t_{2}\right)} \text {. }
$$

- Contribution for $L_{2}$ :

This calculation involves an enumeration of the $T$-equivariant splitting of the normal bundle, similar to the one given above:

$$
\frac{1}{d}\left(t_{1}+t_{2}\right) \cdot \frac{(-1)^{m+d}}{((m-2) !)^{2}(m-1) m} \frac{1}{\tau^{2 m}} \bmod \left(t_{1}+t_{2}\right)^{2} .
$$

- Other curves, nodes, and marked points: The remaining calculations are the same as those given above:

$$
(-1)^{m}((m-1) !)^{2} \tau^{2 m}
$$

The total contribution yields

$$
\begin{aligned}
\left\langle\left[J_{\vec{\rho}}\right]\left|\Theta_{i, j}\right|\left[J_{\vec{\kappa}}\right]\right\rangle & =\sum_{d \geq 1} \frac{1}{d} \frac{(m !)^{2}}{m}(-1)^{m d+m+d} \tau^{2 m}\left(q^{m-1} s_{i} \cdot \ldots \cdot s_{j-1}\right)^{d} \\
& =(-1)^{m-1}\left((n+1) t_{1}\right)^{2 m} \frac{(m !)^{2}}{m} \log \left(1-(-q)^{m-1} s_{i} \cdot \ldots \cdot s_{j-1}\right) .
\end{aligned}
$$




\section{Operator CAlculations}

In this section, we prove the results of the last section for the operator $\Omega_{+}$. The results of the last section give an inductive algorithm (Propositions 4.1] 4.3) which uniquely determines the operator $\Theta_{+}$in terms of

(1) the factorization statement of Proposition 3.2.

(2) the vanishing statement of Proposition 4.9.

(3) the calculation of two-point correlators $\bmod \left(t_{1}+t_{2}\right)^{2}$ in Proposition 4.3 ,

(4) the statement that the coefficients of $\left\langle\prod \mu_{i}\left(\omega_{i}\right)\left|\Theta_{+}\right| \prod \nu_{i}\left(\omega_{i}\right)\right\rangle$ are linear polynomials, proportional to $\left(t_{1}+t_{2}\right)$,

(5) and the vanishing vacuum expectation $\left\langle v_{\emptyset}\left|\Theta_{+}\right| v_{\emptyset}\right\rangle$.

All the other manipulations of the last section involved moving between the Nakajima and fixed-point bases. If we prove each of these statements for $\left(t_{1}+t_{2}\right) \Omega_{+}$, then this inductive algorithm forces

$$
\left(t_{1}+t_{2}\right) \Omega_{+}=\Theta_{+}
$$

It is clear from its definition that $\Omega_{+}$has the same root dependence and degree scaling properties as those of $\Theta_{+}$proved in Proposition 4.4. It therefore suffices to isolate the contribution of a fixed root $\alpha=\alpha_{i, j}$ or, equivalently, to study the coefficient of $s_{i} \cdot \ldots \cdot s_{j-1}$ :

$$
\mathcal{E}_{\alpha}(q)=-\sum_{k \in \mathbb{Z}}: e_{j, i}(k) e_{i, j}(-k):(-q)^{k}
$$

where we have dropped the factor of $t_{1}+t_{2}$ for convenience. The vanishing vacuum expectation is immediate from this formula.

5.1. Commutation relations. We first write the commutation relations for $\mathcal{E}_{\alpha}(q)$ with the Nakajima operators $\mathfrak{p}_{r}(\gamma)$ for $r \neq 0$. Define operators

$$
\mathcal{E}_{\alpha}^{r}(q)=-\sum_{k \in \mathbb{Z}} e_{j, i}(k) e_{i, j}(r-k)(-q)^{k-\frac{r}{2}} .
$$

Notice that, on any fixed graded piece of Fock space, the summands in the above expression vanish for $k$ sufficiently negative. For $r=0$, the discrepancy between this operator and $\mathcal{E}_{\alpha}$ arises from the normal ordering. This can be written as

$$
\mathcal{E}_{\alpha}^{0}(q)=\mathcal{E}_{\alpha}(q)+\left(e_{i i}(0)-e_{j j}(0)\right) \frac{-q}{1+q}+\frac{q}{(1+q)^{2}} \cdot c .
$$

In particular, we have

$$
\mathcal{E}_{\alpha}^{0}(q) v_{\emptyset}=\frac{q}{(1+q)^{2}} v_{\emptyset}
$$

It follows directly from the embedding of the Heisenberg algebra into $\widehat{\mathfrak{g}}$ and from the commutation relations that

$$
\left[\mathfrak{p}_{r}(\gamma), \mathcal{E}_{\alpha}^{s}(q)\right]=(\alpha, \gamma)\left((-q)^{-r / 2}-(-q)^{r / 2}\right) \cdot \mathcal{E}_{\alpha}^{r+s}(q)
$$

where $(\alpha, \gamma)$ denotes the Poincaré pairing on $H_{T}^{*}\left(\mathcal{A}_{n}, \mathbb{Q}\right)$.

This result makes calculating matrix elements with respect to the Nakajima basis extremely simple. In particular, we can easily deduce the factorization statement.

Proposition 5.1. In terms of the Nakajima basis with respect to $\left\{1, \omega_{1}, \ldots, \omega_{n}\right\}$, we have

$$
\left\langle\mu(1) \prod \lambda_{i}\left(\omega_{i}\right)\left|\mathcal{E}_{\alpha}(q)\right| \nu(1) \prod \rho_{i}\left(\omega_{i}\right)\right\rangle=\langle\mu(1) \mid \nu(1)\rangle \cdot\left\langle\lambda_{i}\left(\omega_{i}\right)\left|\mathcal{E}_{\alpha}(q)\right| \rho_{i}\left(\omega_{i}\right)\right\rangle .
$$


Proof. This follows immediately from the fact that

$$
\left[\mathcal{E}_{\alpha}(q), \mathfrak{p}_{r}(1)\right]=0,
$$

which in turn follows from the vanishing

$$
(\alpha, 1)=0 .
$$

Furthermore, it is clear that matrix elements $\left\langle\prod \lambda_{i}\left(\omega_{i}\right)\left|\mathcal{E}_{\alpha}(q)\right| \prod \rho_{i}\left(\omega_{i}\right)\right\rangle$ are nonequivariant constants, so that the coefficients of $\left(t_{1}+t_{2}\right) \Omega_{+}$are linear. All that remains are the vanishing statement and the exact evaluations.

5.2. Diagonalization. We now analyze the matrix elements of $\mathcal{E}_{\alpha}^{0}(q)$ with respect to the fixed-point basis. For our purposes, we only need to understand a certain minor of this matrix $\bmod \left(t_{1}+t_{2}\right)$. More precisely, since all operators and bases are defined over $R=\mathbb{Q}\left[t_{1}, t_{2}\right]_{\left(t_{1}+t_{2}\right)}$, it makes sense to study the reduction of these operators after tensoring with

$$
R /\left(t_{1}+t_{2}\right)=\mathbb{Q}(\tau),
$$

where $\tau \equiv(n+1) t_{1} \bmod t_{1}+t_{2}$.

Recall the tensor product decomposition

$$
\mathcal{F}_{\mathcal{A}_{n}} \otimes R=\bigotimes_{k=1}^{n+1} \mathcal{F}_{\mathbb{C}^{2}, k} \otimes R
$$

where the factors correspond to the $T$-fixed points $p_{1}, \ldots, p_{n+1}$ of $\mathcal{A}_{n}$. Since

$$
\left(\alpha,\left[p_{k}\right]\right)=0
$$

for $k \neq i, j$, we have

$$
\left[\mathcal{E}_{\alpha}^{0}, \mathfrak{p}_{r}\left(\left[p_{k}\right]\right)\right]=0
$$

for $k \neq i, j$. Therefore, this operator admits a decomposition

$$
\mathcal{E}_{\alpha}^{0}(q)=\mathcal{E}_{\alpha}^{\prime}(q) \otimes \mathrm{Id},
$$

where $\mathcal{E}_{\alpha}^{\prime}(q)$ is an operator on

$$
\mathcal{F}_{i, j} \otimes R=\left(\mathcal{F}_{\mathbb{C}^{2}, i} \otimes \mathcal{F}_{\mathbb{C}^{2}, j}\right) \otimes R
$$

and the identity matrix acts on the remaining factors. From now on, we suppress the $\mathbb{C}^{2}$ in our notation. There is a bi-grading on the above space given by

$$
\mathcal{F}_{i, j}=\bigoplus_{a, b \geq 0} \mathcal{F}_{i}^{(a)} \otimes \mathcal{F}_{j}^{(b)} .
$$

We are interested in the minors with respect to this decomposition. That is, if $\pi_{a, b}$ denote the orthogonal projections with respect to this grading, then

$$
\mathrm{M}_{\alpha}(q)=\bigoplus_{a, b \geq 0} \pi_{a, b} \circ \mathcal{E}_{\alpha}^{\prime}(q) \circ \pi_{a, b}
$$

is the operator defined by restricting to the minors where the number of points concentrated at each fixed point is held constant. We want to diagonalize the 
operator

$$
\overline{\mathrm{M}_{\alpha}}(q)
$$

on

$$
\mathcal{F}_{i, j} \otimes R /\left(t_{1}+t_{2}\right)=\mathcal{F}_{i, j} \otimes \mathbb{Q}(\tau)
$$

obtained by an extension of scalars.

The advantage of composing with the projectors $\pi_{a, b}$ is that we have a further factorization of $\overline{\mathrm{M}_{\alpha}}(q)$. It will again be convenient to write everything in terms of symmetric function notation for elements of $\mathcal{F}=\mathcal{F}_{\mathbb{C}^{2}} \otimes \mathbb{Q}(\tau)$.

Let $\mathrm{A}(q)$ be the operator on symmetric functions defined by

$$
\mathrm{A}(q) \cdot \mathrm{p}_{\mu}(z)=\sum_{\nu} \mathrm{p}_{\nu}(z)\left(\sum_{\rho \subset \mu \cap \nu} \frac{1}{\mathfrak{z}(\nu \backslash \rho)} \cdot \frac{(-q)^{1 / 2}}{(1+q)} \cdot f_{\mu \backslash \rho}(q) f_{\nu \backslash \rho}(q)\right) .
$$

In the above expression, $f_{\lambda}(q)$ is defined by

$$
f_{\lambda}(q)=\prod_{i}\left((-q)^{\lambda_{i} / 2}-(-q)^{-\lambda_{i} / 2}\right) .
$$

If $\omega$ is the involution on symmetric functions defined by

$$
\omega\left(\mathrm{p}_{\mu}(z)\right)=(-1)^{l(\mu)} \mathrm{p}_{\mu}(z),
$$

then we also have the conjugate operator

$$
\mathrm{B}(q)=\omega \circ \mathrm{A}(q) \circ \omega .
$$

We then have the decomposition:

\section{Lemma 5.2.}

$$
\overline{\mathrm{M}_{\alpha}}(q)=-\mathrm{B}(q) \otimes \mathrm{A}(q) .
$$

Proof. This follows from the formulas for $\mathrm{A}(q)$ and $\mathrm{B}(q)$ and the commutation relations for $\overline{\mathrm{M}_{\alpha}}(q)$ with respect to the Nakajima operators.

The following lemma is proven in OP3. In what follows, given a partition $\lambda$, we define the function

$$
e(\lambda, q)=\sum_{i}(-q)^{\lambda_{i}-i+\frac{1}{2}}
$$

which is a rational function in $(-q)^{1 / 2}$.

Lemma 5.3. The operators $\mathrm{A}(q)$ and $\mathrm{B}(q)$ are diagonalizable with eigenvectors given by Schur polynomials $\mathbf{s}_{\lambda}(z)$ and eigenvalues $e(\lambda, q)$ and $e\left(\lambda^{\prime}, q\right)$, respectively.

Proof. In OP3, the Gromov-Witten theory of $\mathbf{P}^{1}$ is given in terms of an operator $\mathcal{E}_{\mathbf{P}^{1}}^{0}(z)$ on the space of symmetric functions (identified with standard Fock space). Its eigenvectors are given by Schur functions $\mathrm{s}_{\lambda}(z)$ with eigenvalues $e\left(\lambda,-e^{z}\right)$. Moreover, a direct comparison of the commutation relations with the Nakajima operators shows that

$$
\mathrm{A}\left(e^{z}\right)=\mathcal{E}_{\mathbf{P}^{1}}^{0}(z)
$$

The statement for $\mathrm{B}(q)$ follows from the fact that

$$
\omega\left(s_{\lambda}\right)=s_{\lambda^{\prime}} .
$$

The following proposition, including a vanishing statement, is an immediate corollary of the above discussion. 
Proposition 5.4. The matrix $\overline{\mathrm{M}_{\alpha}}(q)$ is diagonal with respect to the basis

$$
\mathrm{J}_{\lambda}(z) \otimes \mathrm{J}_{\rho}(z)
$$

with eigenvalues

$$
-e_{\lambda^{\prime}}(q) \cdot e_{\rho}(q) .
$$

Furthermore, given two distinct multipartitions $\vec{\lambda} \neq \vec{\eta}$ such that either $\left|\lambda_{i}\right|=\left|\eta_{i}\right|$ or $\left|\lambda_{j}\right|=\left|\eta_{j}\right|$, then

$$
\left\langle\left[J_{\vec{\lambda}}\right]\left|\mathcal{E}_{\alpha}(q)\right|\left[J_{\vec{\eta}}\right]\right\rangle=0 \bmod \left(t_{1}+t_{2}\right) .
$$

5.3. Two-point correlators. We now prove a version of Proposition 4.3 for $\Omega_{+}(q)$. Let Let $\Omega_{[i, j]}\left(q, s_{i}, \ldots, s_{j-1}\right)$ denote the contribution to $\Omega_{+}$arising from monomials in $s_{i}, \ldots, s_{j-1}$ (or, equivalently, curve classes supported in $[i, j]$ ). Recall the multipartitions $\vec{\rho}, \vec{\kappa}, \vec{\theta}, \vec{\sigma}$ from the statement of Proposition 4.2 .

Proposition 5.5. We have the following evaluations for two-point correlators modulo $\left(t_{1}+t_{2}\right)$ :

$$
\begin{aligned}
\left\langle\left[J_{\vec{\rho}}\right]\left|\Omega_{[i, j]}\right|\left[J_{\vec{k}}\right]\right\rangle & =(-1)^{m-1}\left((n+1) t_{1}\right)^{2 m} \frac{(m !)^{2}}{m} \log \left(1-(-q)^{m-1} s_{i j}\right), \\
\left\langle\left[J_{\vec{\theta}}\right]\left|\Omega_{[i, j]}\right|\left[J_{\vec{\sigma}}\right]\right\rangle & =(-1)^{m-1}\left((n+1) t_{1}\right)^{2 m} \frac{(m !)^{2}}{m} \log \left(1-(-q)^{-m+1} s_{i j}\right) .
\end{aligned}
$$

Proof. This immediately reduces to a claim regarding $\overline{\mathcal{E}_{\alpha}}(q)$. Using the commutation relations with Nakajima operators, it suffices to restrict to the case of $n=1$. If we write everything in terms of symmetric functions, the first equality is equivalent to

$$
\left\langle\mathrm{s}_{(m)}(z) \otimes 1\left|\overline{\mathcal{E}_{\alpha}(q)}\right| \mathbf{s}_{(m-1)}(z) \otimes \mathbf{s}_{1}(z)\right\rangle=(-1)^{m}(-q)^{m-1} .
$$

This identity then follows from the eigenvalue calculations of the last section:

$$
\begin{array}{r}
\left\langle\mathbf{s}_{(m)}(z) \otimes 1\left|\overline{\mathcal{E}_{\alpha}(q)}\right| \mathbf{s}_{(m-1)}(z) \otimes \mathbf{s}_{1}(z)\right\rangle=\left\langle\mathbf{s}_{(m)}(z) \otimes 1\left|\overline{\mathcal{E}_{\alpha}(q)}\right| \mathfrak{p}_{-1}(1) \mathbf{s}_{(m-1)}(z) \otimes 1\right\rangle \\
-\left\langle\mathbf{s}_{(m)}(z) \otimes 1\left|\overline{\mathcal{E}_{\alpha}(q)}\right|\left(\mathbf{s}_{(m-1)}(z) \cdot \mathbf{s}_{1}(z)\right) \otimes 1\right\rangle=\left\langle\mathfrak{p}_{-1}^{*}(1) \mathbf{s}_{(m)}(z) \otimes 1\left|\overline{\mathcal{E}_{\alpha}(q)}\right| \mathbf{s}_{(m-1)}(z) \otimes 1\right\rangle \\
-\left\langle\mathbf{s}_{(m)}(z) \otimes 1\left|\overline{\mathcal{E}_{\alpha}(q)}\right|\left(\mathbf{s}_{(m)}(z)+\mathbf{s}_{(m-1,1)}(z)\right) \otimes 1\right\rangle=\left\langle\mathbf{s}_{(m-1)}(z) \otimes 1\left|\overline{\mathcal{E}_{\alpha}(q)}\right| \mathbf{s}_{(m-1)}(z) \otimes 1\right\rangle \\
+\left\langle\mathbf{s}_{(m)}(z) \otimes 1\left|\overline{\mathcal{E}_{\alpha}(q)}\right| \mathbf{s}_{(m)}(z) \otimes 1\right\rangle=(-1)^{m-1} e(\emptyset, q)(e((m-1), q)-e((m), q)) \\
=-(-1)^{m-1}(-q)^{m-1} .
\end{array}
$$

For the second equality, we replace the partition $(m)$ with $\left(1^{m}\right)$, which is equivalent to replacing $q$ with $1 / q$.

\section{Proofs of Main Results}

6.1. Punctual contribution. In this section, we explain how to identify the punctual contributions of the operators $\Theta$ and $\Omega$, that is, to show $\left(t_{1}+t_{2}\right) \Omega_{0}(q)=\Theta_{0}(q)$. This contribution is expressed in terms of the two-point operator for $\operatorname{Hilb}\left(\mathbb{C}^{2}\right)$. In fact, Kiem and Li $\left[\mathrm{KL}\right.$. have used the case of $\mathbb{C}^{2}$ to calculate the punctual two-point operator for an arbitrary surface $S$ using a more general version of the reduced class arguments we make here. However, since we need an equivariant statement, we argue directly. 
We first rewrite $\Omega_{0}(q)$ in terms of the identification $\mathcal{F}_{\mathcal{A}_{n}} \otimes \mathbb{Q}\left(t_{1}, t_{2}\right)=\otimes \mathcal{F}_{\mathbb{C}^{2}} \otimes$ $\mathbb{Q}\left(t_{1}, t_{2}\right)$. It follows from the definition that

$$
\begin{aligned}
\left(t_{1}+t_{2}\right) \Omega_{0}(q) & =-\left(t_{1}+t_{2}\right) \sum_{i=1}^{n+1} \sum_{k \geq 1} \frac{1}{w_{L}^{i} w_{R}^{i}} \mathfrak{p}_{-k}\left(\left[p_{i}\right]\right) \mathfrak{p}_{k}\left(\left[p_{i}\right]\right) \log \left(\frac{1-(-q)^{k}}{1-(-q)}\right) \\
& =\sum_{i=1}^{n+1} \Theta_{\mathbf{C}^{2}, i}(q)
\end{aligned}
$$

where the last equality denotes the two-point operator for $\operatorname{Hilb}\left(\mathbb{C}^{2}\right)$ acting on the $i$-th factor of the tensor product decomposition, as calculated in OP1. Our goal is to prove the same statement for $\Theta_{0}(q)$.

The first step is to apply the reduced class construction to prove an analog of the factorization statement.

Proposition 6.1. We have the following two identities for insertions labelled with 1 :

$$
\begin{aligned}
\left\langle\mu(1) \prod \lambda_{i}\left(\omega_{i}\right)\left|\Theta_{0}\right| \nu(1) \prod \rho_{i}\left(\omega_{i}\right)\right\rangle= & \left\langle\mu(1)\left|\Theta_{0}\right| \nu(1)\right\rangle \cdot\left\langle\prod \lambda_{i}\left(\omega_{i}\right) \mid \prod \rho_{i}\left(\omega_{i}\right)\right\rangle \\
& +\langle\mu(1) \mid \nu(1)\rangle \cdot\left\langle\prod \lambda_{i}\left(\omega_{i}\right)\left|\Theta_{0}(q)\right| \prod \rho_{i}\left(\omega_{i}\right)\right\rangle, \\
\left\langle\mu(1)\left|\Theta_{0}(q)\right| \nu(1)\right\rangle= & \left(t_{1}+t_{2}\right)\left\langle\mu(1)\left|\Omega_{0}(q)\right| \nu(1)\right\rangle .
\end{aligned}
$$

Proof. For the first claim, we proceed exactly as in the proof of Proposition 3.2 If $l(\mu) \geq l(\nu)$, we expand the cohomology class 1 in terms of fixed points $\left[p_{i}\right]$. The same dimension analysis forces $l(\mu)=l(\nu)$ and in fact $\mu=\nu$. Moreover, we again rewrite the invariant (up to a factor of $\left(t_{1}+t_{2}\right)$ ) as a nonequivariant reduced invariant

$$
\left(\mu(1) \prod \lambda_{i}\left(\omega_{i}\right), \nu([p]) \prod \rho_{i}\left(\omega_{i}\right)\right)^{\mathrm{red}},
$$

where $p$ is a point on $\mathcal{A}_{n}$ away from the exceptional locus. Again, if the curve class is not contracted over both $p$ and the exceptional locus, there is a second trivial factor to the obstruction theory that forces vanishing. The only difference is that both possibilities can now occur, since we have a punctual curve class. They give the two contributions on the right-hand side.

For the second claim, we use the expression for $\Omega_{0}(q)$ from equation (6). Again, it suffices to prove the claim after expanding 1 in terms of fixed points in the insertions associated to $\nu$. The same nonequivariant-reduced argument shows that

$$
\frac{\left\langle\mu(1)\left|\Theta_{0}(q)\right| \prod \nu_{i}\left(\left[p_{i}\right]\right)\right\rangle}{\left\langle\mu(1) \mid \prod \nu_{i}\left(\left[p_{i}\right]\right)\right\rangle}=\sum_{i=1}^{n+1} \frac{\left\langle\mu(1)\left|\Theta_{0}(q)\right| \nu_{i}\left(\left[p_{i}\right]\right)\right\rangle}{\left\langle\mu(1) \mid \nu_{i}\left(\left[p_{i}\right]\right)\right\rangle} .
$$

The analogous equality is trivially true for $\Omega_{0}(q)$ using the tensor-product decomposition of (6). This reduces the claim to the invariant $\left\langle\mu(1)\left|\Theta_{0}(q)\right| \mu\left(\left[p_{i}\right]\right)\right\rangle$ for fixed $i$. Since everything is concentrated at a single fixed point, we can directly identify the invariant with a calculation on $\mathbb{C}^{2}$ to give

$$
\left\langle\mu(1)\left|\Theta_{0}(q)\right| \mu\left(\left[p_{i}\right]\right)\right\rangle=\left\langle\mu(1)\left|\Theta_{\mathbf{C}^{2}, i}(q)\right| \mu\left(\left[p_{i}\right]\right)\right\rangle=\left(t_{1}+t_{2}\right)\left\langle\mu(1)\left|\Omega_{0}(q)\right| \mu\left(\left[p_{i}\right]\right)\right\rangle .
$$




\section{Proposition 6.2.}

$$
\begin{aligned}
\left\langle\mu(1) \prod \lambda_{i}\left(\omega_{i}\right)\left|\Omega_{0}\right| \nu(1) \prod \rho_{i}\left(\omega_{i}\right)\right\rangle & =\left\langle\mu(1)\left|\Omega_{0}\right| \nu(1)\right\rangle \cdot\left\langle\prod \lambda_{i}\left(\omega_{i}\right) \mid \prod \rho_{i}\left(\omega_{i}\right)\right\rangle \\
& +\langle\mu(1) \mid \nu(1)\rangle \cdot\left\langle\prod \lambda_{i}\left(\omega_{i}\right)\left|\Omega_{0}(q)\right| \prod \rho_{i}\left(\omega_{i}\right)\right\rangle .
\end{aligned}
$$

Proof. This follows immediately from the fact that the operators $\mathfrak{p}_{k}(1)$ and $\mathfrak{p}_{k}\left(\omega_{i}\right)$ commute.

The main proposition now follows quite easily.

\section{Proposition 6.3.}

$$
\Theta_{0}(q)=\left(t_{1}+t_{2}\right) \Omega_{0}(q) .
$$

Proof. We proceed by induction on the number of points $m$. We first observe that if either $\vec{\mu}$ or $\vec{\nu}$ contains a part labelled by 1 , then Propositions 6.1 and 6.2 along with the inductive hypothesis establish the equality.

We work in the Nakajima basis with respect to the fixed points $p_{i}$. Given multipartitions $\vec{\mu}, \vec{\nu}$, there is a trivial vanishing

$$
\left\langle\prod \mu_{i}\left(\left[p_{i}\right]\right)\left|\Theta_{0}(q)\right| \prod \nu_{i}\left(\left[p_{i}\right]\right)\right\rangle=\left\langle\prod \mu_{i}\left(\left[p_{i}\right]\right)\left|\Omega_{0}(q)\right| \prod \nu_{i}\left(\left[p_{i}\right]\right)\right\rangle=0
$$

if there exists an $i$ for which

$$
\left|\mu_{i}\right| \neq\left|\nu_{i}\right|
$$

For $\Omega_{0}(q)$ this follows from the tensor-product decomposition; for $\Theta(q)$ this is because the number of points concentrated at each fixed point must be constant for punctual curves. In the general case, if we assume $\mu_{1} \neq \emptyset$, we expand

$$
\left[p_{1}\right]=c_{0} \cdot 1+c_{2}\left[p_{2}\right]+\cdots+c_{n+1}\left[p_{n+1}\right]
$$

for the insertions of $\mu_{1}$. The terms corresponding to $p_{2}, \ldots, p_{n+1}$ cannot contribute by the same trivial vanishing statement. We thus have

$$
\left\langle\prod \mu_{i}\left(\left[p_{i}\right]\right)\left|\Theta_{0}(q)\right| \prod \nu_{i}\left(\left[p_{i}\right]\right)\right\rangle=\left\langle\mu_{1}\left(c_{0} \cdot 1\right) \prod \mu_{i}\left(\left[p_{i}\right]\right)\left|\Theta_{0}(q)\right| \prod \nu_{i}\left(\left[p_{i}\right]\right)\right\rangle
$$

and the analogous equality for $\Omega_{0}(q)$. The claim then follows from our initial observation.

6.2. Proof of Theorem 2.1. For the nonpunctual contributions, the equality

$$
\Theta_{+}=\left(t_{1}+t_{2}\right) \Omega_{+}
$$

follows from the arguments of Sections 4 and 5 More precisely, we have shown in these sections that both operators satisfy factorization and vanishing statements. These are Propositions 3.2 and 4.9 for $\Theta_{+}$and Propositions 5.1 and 5.4 for $\Omega_{+}$. The algorithm in Section 4 explains how these propositions reduce the calculation of each operator to the precise calculations of Propositions 4.3 and 5.5 where equality is proven directly. For the punctual contribution, the equality

$$
\Theta_{0}=\left(t_{1}+t_{2}\right) \Omega_{0}
$$

is Proposition 6.3. 
6.3. Generation conjecture. Recall that Corollary 2.2 gives the operators for quantum multiplication by divisors $D$ and $\left(1, \omega_{i}\right)$ :

$$
\begin{gathered}
M_{D}=M_{D}^{c l}+\left(t_{1}+t_{2}\right) q \frac{d}{d q} \Omega\left(q, s_{1}, \ldots, s_{n}\right), \\
M_{\left(1, \omega_{i}\right)}=M_{\left(1, \omega_{i}\right)}^{c l}+\left(t_{1}+t_{2}\right) s_{i} \frac{d}{d s_{i}} \Omega_{+}\left(q, s_{1}, \ldots, s_{n}\right) .
\end{gathered}
$$

Since quantum cohomology defines a graded commutative ring, these operators commute. It therefore makes sense to discuss the joint spectrum of these operators. We conjecture the following nondegeneracy statement for this spectrum.

Conjecture. The joint eigenspaces for the operators $M_{D}, M_{\left(1, \omega_{i}\right)}$ are one-dimensional for all $m>0$.

Under the assumption of this conjecture 11 we can immediately generate the full quantum ring as well as all genus 0 invariants.

Corollary* 6.4. Assuming the above conjecture, the divisors $D$ and $\left(1, \omega_{i}\right)$ generate the small quantum cohomology ring $H_{T}^{*}\left(\mathcal{A}_{n}, \mathbb{Q}\right)$. Moreover, we can calculate an arbitrary k-point genus 0 Gromov-Witten invariant for $\operatorname{Hilb}\left(\mathcal{A}_{n}\right)$ in terms of these operators.

Proof. The quantum cohomology ring $H_{T}^{*}\left(\mathcal{A}_{n}, \mathbb{Q}\right) \otimes \mathbb{Q}\left(q, s_{1}, \ldots, s_{n}\right)$ is semisimple since it is a deformation of the semisimple classical equivariant cohomology. The idempotents are simultaneous eigenvectors for $M_{D}, M_{\left(1, \omega_{i}\right)}$. Given the nondegeneracy conjecture, a Vandermonde argument shows that the vectors

$$
M_{D}^{a} \prod_{i} M_{\left(1, \omega_{i}\right)}^{b_{i}}\left(1^{m}\right), \quad a, b_{i} \geq 0,
$$

span the full cohomology ring. The second statement follows from standard reconstruction statements for varieties whose small quantum cohomology ring is generated by divisors ([KM, OP1]).

While we are unable to prove our nondegeneracy conjecture, we can show the following partial result.

Proposition 6.5. The operator for the purely quantum contribution

$$
M_{D}\left(q, s_{1}, \ldots, s_{n}\right)-M_{D}^{c l}=\left(t_{1}+t_{2}\right) q \frac{d}{d q} \Omega\left(q, s_{1}, \ldots, s_{n}\right)
$$

has distinct eigenvalues.

Proof. We will sketch the proof for $\mathcal{A}_{1}$; the same argument works in general. In order to show that $q \frac{d}{d q} \Omega(q, s)$ has distinct eigenvalues, we write our operator as a power series in $s$ and apply a perturbation theory argument. That is, we write

$$
q \frac{d}{d q} \Omega(q, s)=q \frac{d}{d q} \Omega_{0}(q)+s q \frac{d}{d q} \mathcal{E}(q)+O\left(s^{2}\right)
$$

and study the restriction of $q \frac{d}{d q} \mathcal{E}(q)$ to the degenerate eigenspaces of the punctual operator $\Omega_{0}(q)$. From the explicit form of $\Omega_{0}(q)$, we know that an eigenbasis is

\footnotetext{
${ }^{1}$ Statements that rely on this conjecture will be denoted with an asterisk.
} 
given by the Nakajima basis with fixed-point insertions; for every partition $\mu$ of $m$, we have a degenerate eigenspace

$$
V_{\mu}=\operatorname{Span}\left\{\mu_{1}\left(\left[p_{1}\right]\right) \mu_{2}\left(\left[p_{2}\right]\right) \mid \mu_{1}+\mu_{2}=\mu\right\} .
$$

It suffices to show that the restriction and projection of $q \frac{d}{d q} \mathcal{E}(q)$ to each $V_{\mu}$ has distinct eigenvalues $\mathrm{K}$.

Let $r_{k}$ denote the multiplicity of $k$ as a part of $\mu$. Then the eigenspace $V_{\mu}$ admits a tensor product decomposition:

$$
V_{\mu}=\otimes_{k} S^{r_{k}}\left(V_{(k)}\right) .
$$

Moreover, the first-order perturbation also admits a factorization

$$
\left.\frac{(1+q)^{2}}{q} \mathcal{E}_{0}(q)\right|_{V_{\mu}}=\otimes S^{r_{k}}\left(\left.\frac{(1+q)^{2}}{q} \mathcal{E}_{0}(q)\right|_{V_{(k)}}\right) \text {. }
$$

For each fixed part $k$, the eigenvectors of $\left.\frac{(1+q)^{2}}{q} \mathcal{E}_{0}(q)\right|_{V_{(k)}}$ are easily seen to be

$$
\frac{1}{2 t_{1}} k\left(\left[p_{1}\right]\right)-\frac{1}{2 t_{2}} k\left(\left[p_{2}\right]\right), \frac{1}{t_{2}-t_{1}} k\left(\left[p_{1}\right]\right)+\frac{1}{t_{1}-t_{2}} k\left(\left[p_{2}\right]\right)
$$

with eigenvalues given by

$$
1,1+\frac{2}{k}\left((-q)^{k / 2}-(-q)^{-k / 2}\right)^{2}
$$

respectively. The eigenvectors and eigenvalues in the general case are obtained by multiplying these eigenvalues over the distinct parts of $\mu$. The eigenvalues are given by

$$
\prod_{k}\left(1+\frac{2}{k}\left((-q)^{k / 2}-(-q)^{-k / 2}\right)^{2}\right)^{s_{k}}, \quad 0 \leq s_{k}<r_{k},
$$

and are clearly distinct for different values of $s_{k}$. Finally, the eigenvalues for the actual perturbation are obtained by taking the derivative of the above expression and are again clearly distinct.

We consider this proposition good evidence for the generation conjecture since we are taking an operator-valued function of $q$ and $s$ with nondegenerate spectrum and adding an operator with no $q, s$-dependence. Unfortunately, this fact alone is not sufficient to prove the claim. In the case of $\mathbb{C}^{2}$ where the purely quantum part of the operator again has nondegenerate spectrum, it is possible to find a limit in the equivariant parameters in which the classical contribution is suppressed. However, a similar argument does not seem possible in our case.

6.4. Comparison with Gromov-Witten theory of $\mathcal{A}_{n} \times \mathbf{P}^{1}$. Here we follow the notation from Section 1.3 and Section 4.1 of [M]. The generating function

$$
\mathrm{Z}_{G W}^{\prime}\left(\mathcal{A}_{n} \times \mathbf{P}^{1}\right)_{\vec{\mu}, \vec{\nu}, \vec{\rho}}
$$

encodes the all-genus Gromov-Witten theory of $\mathcal{A}_{n} \times \mathbf{P}^{1}$ with relative conditions given by the cohomology-weighted partitions $\vec{\mu}, \vec{\nu}, \vec{\rho}$. In $[\mathrm{M}]$, it is explained how to calculate these invariants when one of these relative conditions corresponds to a divisor insertion. By comparing with our calculations here, we have the following proposition. 
Proposition 6.6. Under the variable substitution $q=-e^{i u}$, we have

$$
(-1)^{m}\langle\vec{\mu},(2), \vec{\nu}\rangle_{\mathcal{A}_{n}}^{\mathrm{Hilb}}=(-i u)^{-1+l(\mu)+l(\nu)} \mathbf{Z}^{\prime}\left(\mathcal{A}_{n} \times \mathbf{P}^{1}\right)_{\vec{\mu},(2), \vec{\nu}}
$$

and

$$
(-1)^{m}\left\langle\vec{\mu},\left(1, \omega_{k}\right), \vec{\nu}\right\rangle_{\mathcal{A}_{n}}^{\mathrm{Hilb}}=(-i u)^{l(\mu)+l(\nu)} \mathbf{Z}^{\prime}\left(\mathcal{A}_{n} \times \mathbf{P}^{1}\right)_{\vec{\mu},\left(1, \omega_{k}\right), \vec{\nu}}
$$

Proof. Note that the substitution makes sense because our three-point functions are rational functions of $q$. In $[\mathrm{M}$, an analog of the root dependence and degree scaling statement are proven; it therefore suffices to consider $\mathcal{A}_{1}$ and the coefficient of $s^{1}$. In that case, we can compare $\Omega_{+}(q, s)$ with the expression $\Theta^{\bullet}(u, s)$ from that paper.

There is a direct geometric relationship between the relative Gromov-Witten theory of $\mathcal{A}_{1} \times \mathbf{P}^{1}$ and the stationary theory of $\mathbf{P}^{1}$. This explains why the operator controlling this latter theory played a role in Section 5.2 .

We can use the all-genus relative Gromov-Witten theory of $\mathcal{A}_{n} \times \mathbf{P}^{1}$ to define a new ring deformation of $H_{T}^{*}\left(\operatorname{Hilb}\left(\mathcal{A}_{n}\right), \mathbb{Q}\right)$ over the ring

$$
R^{\mathrm{GW}}=\mathbb{Q}\left(t_{1}, t_{2}\right)((u))\left[\left[s_{1}, \ldots, s_{n}\right]\right] .
$$

Given three cohomology-weighted partitions $\vec{\mu}, \vec{\nu}, \vec{\rho}$ of $m$, we define a product $*$ using the following structure constants:

$$
\langle\vec{\mu}, \vec{\nu} * \vec{\rho}\rangle=(-i u)^{-m+l(\mu)+l(\nu)+l(\rho)} \mathbf{Z}^{\prime}\left(\mathcal{A}_{n} \times \mathbf{P}^{1}\right) \vec{\mu}, \vec{\nu}, \vec{\rho} .
$$

The following proposition is established in $[\mathrm{M}]$.

Proposition 6.7. The $R^{\mathrm{GW}}$-module $H_{T}^{*}\left(\operatorname{Hilb}\left(\mathcal{A}_{n}\right), \mathbb{Q}\right) \otimes R^{\mathrm{GW}}$ with the product defined above satisfies the axioms of an $R^{\mathrm{GW}}$-algebra.

In terms of this ring structure, Corollary 2.2 states that we can identify the multiplication operators for $(2)$ and $\left(1, \omega_{i}\right)$. Under the assumption of the generation conjecture, this would imply the following general statement of the GromovWitten/Hilbert correspondence.

Corollary* 6.8. Under the variable substitution $q=-e^{i u}$, we have

$$
\langle\vec{\mu}, \vec{\nu}, \vec{\rho}\rangle_{\mathcal{A}_{n}}^{\mathrm{Hilb}}=(-i u)^{-m+l(\mu)+l(\nu)+l(\rho)} \mathbf{Z}^{\prime}\left(\mathcal{A}_{n} \times \mathbf{P}^{1}\right)_{\vec{\mu}, \vec{\nu}, \vec{\rho}} .
$$

In this form, this statement generalizes the correspondence proven for $\mathbb{C}^{2}$ in $[\mathrm{BP}$, OP1. Heuristically, it states that the two ring deformations defined by quantum cohomology and the relative theory of $\mathcal{A}_{n} \times \mathbf{P}^{1}$ are isomorphic after a specific change of variables.

Both ring structures defined in this correspondence make sense for an arbitrary surface $S$. However, the precise relation given here does not hold in this generality. For instance, in the case of $m=1$, the quantum cohomology structure constants have no $q$-dependence, while the Gromov-Witten theory of $S \times \mathbf{P}^{1}$ in general will have nontrivial $u$-dependence. This is already in the case of $\mathbf{P}^{2}$. The special feature of the geometry that allows us to make such a simple matching is the existence of a holomorphic symplectic form.

Notice that in the ring isomorphism no change of variables is required on the parameters corresponding to curve classes on the surfaces $\mathcal{A}_{n}$. Similarly, the Nakajima basis is identified directly with relative conditions in the Gromov-Witten theory of $\mathcal{A}_{n} \times \mathbf{P}^{1}$. If we weaken this strong constraint on the change of variables, it is reasonable to expect the correspondence to generalize. 


\section{FURTHER DIRECTIONS}

7.1. Quantum differential equation. The quantum differential equation associated to $\operatorname{Hilb}_{m}\left(\mathcal{A}_{n}\right)$ is the system of linear differential equations given by

$$
\left\{\begin{array}{l}
q \frac{\partial}{\partial q} \psi=M_{D} \psi, \\
s_{i} \frac{\partial}{\partial s_{i}} \psi=M_{\left(1, \omega_{i}\right)} \psi,
\end{array} \quad \psi\left(q, s_{i}\right) \in H_{T}^{*}\left(\operatorname{Hilb}_{m}\left(\mathcal{A}_{n}\right)\right)((q))\left[\left[s_{i}\right]\right] .\right.
$$

This system defines a flat connection $\nabla_{\mathcal{A}_{n}}\left(t_{1}, t_{2}\right)$ on $\mathbb{C}^{n+1}$ for the trivial bundle associated to $H^{*}\left(\operatorname{Hilb}_{m}\left(\mathcal{A}_{n}\right), \mathbb{C}\right)$ with regular singularities along the hypersurfaces

$$
\begin{aligned}
(-q)^{k} & =1, \quad k=1, \ldots, m-1, \\
\left(s_{i} \cdot \ldots \cdot s_{j-1}\right)(-q)^{k} & =1, \quad k=-m+1, \ldots, m-1, \quad 1 \leq i<j \leq n+1 .
\end{aligned}
$$

In the case of $\mathbb{C}^{2}$, the quantum differential equation can be viewed as a nonstationary extension of the Calogero-Sutherland system with extremely well-behaved monodromy properties OP1. If we take the differential equation obtained by tensoring $n+1$ copies of this nonstationary system, (7) can be viewed as a deformation of this system in the $s$-variables where the coupling between the different factors is essentially controlled by our operator $\Omega_{+}$. Moreover, as we discuss next, many of the qualitatively nice features of the monodromy of $\nabla_{\mathbb{C}^{2}}$ extend to this deformation.

As an example, in the case of $n=1$ with $m=2$ points, the associated matrices are given as follows. We order the standard basis of $H_{T}\left(\operatorname{Hilb}_{2}\left(\mathcal{A}_{1}\right)\right)$ in the following way:

$$
\frac{\mathfrak{p}_{-1}^{2}(E)}{2} v_{\emptyset}, \quad \frac{\mathfrak{p}_{-2}(E)}{2} v_{\emptyset}, \quad \mathfrak{p}_{-1}(E) \mathfrak{p}_{-1}(1) v_{\emptyset}, \quad \frac{\mathfrak{p}_{-1}^{2}(1)}{2} v_{\emptyset}, \quad \frac{\mathfrak{p}_{-2}(1)}{2} v_{\emptyset}
$$

The notation $\theta=t_{1}+t_{2}$ is used in the formulas for the divisor multiplication operators:

$$
\begin{aligned}
M_{(1, \omega)} & =\left(\begin{array}{ccccc}
\frac{2 \theta s(q+1 / q+2 s)}{(1+s q)(1+s / q)} & \frac{\theta s(1 / q-q)}{(1+s q)(1+s / q)} & -1 & 0 & 0 \\
\frac{2 s(1 / q-q)}{(1+s / q)(1+s / q)} & \frac{\theta q(1+1 / q)^{2}(1+s)}{(1+s q)(1+s / q)(1-s)} & 0 & 0 & -1 \\
2 t_{1} t_{2} & 0 & \frac{\theta(1+s)}{s-1} & -\frac{1}{2} & 0 \\
0 & 0 & t_{1} t_{2} & 2 \theta & 0 \\
0 & 2 t_{1} t_{2} & 0 & 0 & 2 \theta
\end{array}\right), \\
M_{D} & =\left(\begin{array}{ccccc}
\frac{2 \theta(q+1 / q+2 s)}{(1+s q)(1+s / q)} & \frac{\theta\left(1-s^{2}\right)}{(1+s q)(1+s / q)} & 0 & 0 & -\frac{1}{2} \\
\frac{2 \theta\left(1-s^{2}\right)}{(1+s q)(1+s / q)} & \frac{\theta(1+s)^{2}(1+q)}{(1+s q)(1+s / q)(1-q)} & 1 & 0 & 0 \\
0 & -2 t_{1} t_{2} & 0 & 0 & 0 \\
0 & 0 & 0 & 0 & 2 t_{1} t_{2} \\
-4 t_{1} t_{2} & 0 & 0 & -1 & \frac{\theta(1+q)}{1-q}
\end{array}\right) .
\end{aligned}
$$

7.2. Exponents and monodromy. We first calculate the eigenvalues of the residues associated to $\nabla$ around its singularities. Notice in particular that, for integer values of the level $t_{1}+t_{2}$, these eigenvalues are integral. We do not discuss the 
residue along the first group of the singularities (8) because they are studied in OP1]:

Proposition 7.1. The residue of the operator $M_{\left(1, \omega_{i}\right)}$ along the divisor

$$
s^{\alpha}(-q)^{k}=1
$$

is zero if $\left(\alpha, \omega_{i}\right)=0$. In general, it is diagonalizable and:

- There exists $N=N(\alpha, k)$ such that

$$
\operatorname{Spec}\left(\operatorname{Res}_{s^{\alpha}(-q)^{k}=1} M_{\left(1, \omega_{i}\right)}\right) \subset\left\{\left(t_{1}+t_{2}\right) l(k+l-1)\right\}_{l=1, \ldots, N} .
$$

- If $k \neq 0$, then $N(\alpha, k)<m / k$.

For $M_{D}$, we have

$$
\operatorname{Res}_{s^{\alpha}(-q)^{k}=1} M_{D}=\operatorname{RRes}_{s^{\alpha}(-q)^{k}=1} M_{\left(1, \omega_{i}\right)}
$$

for any $i$ such that $\left(\alpha, \omega_{i}\right) \neq 0$.

Proof. If $\left(\alpha, \omega_{i}\right)=0$, the vanishing statement is easy. Otherwise, let us introduce an auxilary operator:

$$
G_{l}=e_{-\alpha}(-k)^{l} e_{\alpha}(k)^{l}, \quad G_{1}=\operatorname{Res}_{s^{\alpha}(-q)^{k}=1} M_{\left(1, \omega_{i}\right)} .
$$

The space $H_{T}\left(H i l b_{m}\left(\mathcal{A}_{n}\right)\right)$ is the weight space $V[\Lambda-m \delta]$ of the highest weight representation. Hence there exists $N=N(\alpha, k)$ such that

$$
e_{\alpha}(k)^{N} V[\Lambda-m \delta]=0 .
$$

By the weight consideration, in the case $k \neq 0$ the number $N$ satisfies the proposed inequality. The following consequence of the relations in the Lie algebra completes the proof:

$$
\left(G_{1}-l(k+l-1)\right) G_{l} v=G_{l+1} v,
$$

for any $v \in V$. The claim on $M_{D}$ is immediate from our formulas.

We sketch the construction of a level-raising operator identifying the monodromies of $\nabla_{\mathcal{A}_{n}}\left(t_{1}, t_{2}\right)$ and $\nabla_{\mathcal{A}_{n}}\left(t_{1}-a, t_{2}-b\right)$ for $a, b \in \mathbb{Z}$. Let $\mathcal{X}(a, b)$ denote the family of $\mathcal{A}_{n}$ surfaces over $\mathbf{P}^{1}$ associated to $\mathcal{O}(a) \oplus \mathcal{O}(b)$ by fiberwise quotient and resolution. We set

$$
\mathrm{S}(a, b)
$$

to be the operator encoding its Gromov-Witten theory relative to the fibers over 0 and $\infty$. Under the assumption of the generation conjecture, $\mathrm{S}(a, b)$ can be computed using the techniques of [M] and is given by rational functions in $q=e^{-i u}, s_{1}, \ldots, s_{n}$. A localization argument (and the GW/Hilb comparison) shows that

$$
\nabla_{\mathcal{A}_{n}}\left(t_{1}, t_{2}\right) \mathrm{S}(a, b)=\mathrm{S}(a, b) \nabla_{\mathcal{A}_{n}}\left(t_{1}-a, t_{2}-b\right)
$$

so that $\mathrm{S}(a, b)$ defines an intertwiner operator, identifying the monodromy provided that $t_{1}, t_{2}$ avoid a finite set of rational numbers which give poles for $\mathrm{S}$.

This generalizes the same construction for $\nabla_{\mathbb{C}^{2}}$ given in OP1]. One can again show that at integer level $t_{1}+t_{2} \in \mathbb{Z}$, the monodromy is abelian and is semisimple provided that $t_{1}$ and $t_{2}$ avoid the singularities of $S$. At these values, the fundamental solution to the QDE is basically given by rational functions of $q, s_{1}, \ldots, s_{n}$. In more 
detail, the fundamental solution to the QDE is of the form

$$
B\left(q, s_{1}, \ldots, s_{n}\right) \cdot q^{M_{D}^{c l}} \prod_{i} s_{i}^{M_{\left(1, \omega_{i}\right)}^{c l}}
$$

where $B\left(q, s_{1}, \ldots, s_{n}\right)$ is a rational function of $q$ and $s_{1}, \ldots, s_{n}$.

7.3. Generalization to $D, E$ resolutions. Finally, we briefly explain how the calculations of this paper can be extended to resolutions $S_{\Gamma}$ of rational surface singularities associated to root lattices $\Gamma$ of types $D$ and $E$. The argument here was suggested to us by Jim Bryan and is based on an argument first used in BKL]. As these singularites are no longer toric, there is only a $\mathbb{C}^{*}$-action on $S_{\Gamma}$.

Dimension considerations reduce the two-point calculation to studying the nonequivariant reduced virtual class. Let $X_{0} \rightarrow \Delta$ be a smooth family of surfaces over the disk $\Delta$, obtained from a map from $\Delta$ to the versal deformation space of $S_{\Gamma}$. Its fiber over the origin is the resolved surface $S_{\Gamma}$, while all other fibers are given by affine surfaces; in particular, all compact curves on $X$ lie over the origin. This family admits a deformation $X_{z} \rightarrow \Delta$ so that for $z \neq 0$, there are a finite number of nonaffine fibers each isomorphic to $\mathcal{A}_{1}$. These nonaffine fibers are in bijection with positive roots $\alpha$ of $\Gamma$, and the smooth rational curve lies in the corresponding curve class $\alpha$.

For both $X_{0}$ and $X_{z}$, we can take associated family of Hilbert schemes

$$
\operatorname{Hilb}\left(X_{0} / \Delta\right), \operatorname{Hilb}\left(X_{z} / \Delta\right) \rightarrow \Delta
$$

which are again deformation equivalent. An effective curve on $\operatorname{Hilb}\left(X_{0} / \Delta\right)$ must be contained in $\operatorname{Hilb}\left(S_{\Gamma}\right) \subset \operatorname{Hilb}\left(X_{0} / \Delta\right)$; similarly, an effective curve on $\operatorname{Hilb}\left(X_{z} / \Delta\right)$ must be contained in one of the copies of $\operatorname{Hilb}\left(\mathcal{A}_{1}\right) \subset \operatorname{Hilb}\left(X_{z} / \Delta\right)$. The key observation is that, for nonpunctual curve classes $\beta$, we can identify the reduced virtual class on $\operatorname{Hilb}\left(S_{\Gamma}\right)$ with the relative virtual class (defined in the usual sense) of the family $\operatorname{Hilb}\left(X_{0} / \Delta\right)$ over $\Delta$ :

$$
\left[\bar{M}_{0,2}\left(\operatorname{Hilb}\left(S_{\Gamma}\right), \beta\right)\right]^{\mathrm{red}}=\left[\bar{M}_{0,2}\left(\operatorname{Hilb}\left(X_{0}, \Delta\right), \beta\right)\right]^{\mathrm{vir}} .
$$

A detailed proof can be found, for instance, in $\mathrm{MP}$. The analogous statement holds for $X_{z}$. Along with deformation invariance between $X_{0}$ and $X_{z}$, this immediately gives that only root curve classes contribute to reduced invariants and, in that case, the calculation is given by the case of $\mathcal{A}_{1}$. The result is that the two-point operator is again given by the same expression in terms of the action of type $D, E$ affine algebras $\widehat{\mathfrak{g}}$ on Fock space:

$$
\Theta_{+}=\sum_{\alpha \in R^{+}} \sum_{k}: e_{\alpha}(k) e_{-\alpha}(-k): \log \left(1-s^{\alpha} q^{k}\right) .
$$

\section{ACKNOWLEDGEMENTS}

We wish to thank A. Okounkov and R. Pandharipande for many conversations about their paper [OP1], which were important in shaping the arguments here. We also thank J. Bryan, P. Etingof, and S. Loktev for many useful discussions. 


\section{REFERENCES}

[B] A. Borel, Algebraic D-modules, Academic Press, Orlando, Florida, 1987. MR0882000 (89g:32014)

[BKL] J. Bryan, S. Katz, N.C. Leung, Multiple covers and the integrality conjecture for rational curves in Calabi-Yau threefolds, J. Algebraic Geom. 10 (3) (2001), 549-568. MR.1832332 (2002j:14047)

[BP] J. Bryan, R. Pandharipande, The local Gromov-Witten theory of curves, JAMS 21 (2008), 101-136. MR2350052 (2008h:14057)

[GP] T. Graber, R. Pandharipande, Localization of virtual classes, Invent. Math. 135 (1999), no. 2, 487-518. MR1666787(2000h:14005)

[Gr] I. Grojnowski, Instantons and affine algebras. I. The Hilbert scheme and vertex operators, Math. Res. Lett. 3 (1996), no. 2, 275-291. MR1386846 (97f:14041)

[K] T. Kato, Perturbation theory for linear operators, Reprint of the 1980 edition. Classics in Mathematics. Springer-Verlag, Berlin, 1995. MR.1335452 (96a:47025)

[KL] Young-Hoon Kiem, Jun Li, Gromov-Witten invariants of varieties with holomorphic 2-forms, arXiv:math/0707.2986.

[KM] M. Kontsevich, Yu. Manin, Gromov-Witten classes, quantum cohomology, and enumerative geometry, Comm. Math. Phys. 164 (1994), no. 3, 525-562. MR1291244(95i:14049)

[LS] M. Lehn, Ch. Sorger, The cup product of Hilbert schemes for $K 3$ surfaces, Invent. Math. 152 (2003), no. 2, 305-329. MR.1974889 (2004a:14004)

[LQW] W. Li, Z. Qin, W. Wang, The cohomology rings of Hilbert schemes via Jack polynomials, CRM Proceedings and Lecture Notes 38 (2004), 249-258. MR2096149 (2005k:14009)

[Man] M. Manetti, Lie description of higher obstructions to deforming submanifolds, Ann. Sc. Norm. Super. Pisa Cl. Sci. (5) 6 (2007), no. 4, 631-659. MR.2394413

[M] D. Maulik, GW theory of $\mathcal{A}_{n}$ resolutions, to appear in Geometry and Topology, arXiv:math/0802.2681.

[MO] D. Maulik, A. Oblomkov, DT theory of $\mathcal{A}_{n} \times \mathbf{P}^{1}$, to appear in Compos. Math., arXiv:math/0802.2739.

[MOOP] D. Maulik, A. Oblomkov, A. Okounkov, R. Pandharipande, GW/DT correspondence for toric threefolds, arXiv:math/0809.3976.

[MP] D. Maulik, R. Pandharipande, Gromov-Witten theory and Noether-Lefschetz theory, arXiv:math/0705.1653.

[N1] H. Nakajima, Heisenberg algebra and Hilbert schemes of points on projective surfaces, Ann. of Math. (2) 145 (1997), no. 2, 379-388. MR1441880 (98h:14006)

[N2] H. Nakajima, Jack polynomials and Hilbert schemes of points on surfaces, 1996, arXiv:math/9610021.

[N3] H. Nakajima, Lectures on Hilbert schemes of points on surfaces, University Lecture Series, 18, American Mathematical Society, Providence, RI, 1999. MR.1711344 (2001b:14007)

[OP1] A. Okounkov, R. Pandharipande, Quantum cohomology of the Hilbert scheme of points in the plane, arXiv:math/0411210.

[OP2] A. Okounkov, R. Pandharipande, The local Donaldson-Thomas theory of curves, arXiv:math/0512573.

[OP3] A. Okounkov, R. Pandharipande, Gromov-Witten theory, Hurwitz theory, and completed cycles, Ann. of Math. (2) 163 (2006), no. 2, 517-560. MR2199225 (2007b:14123)

[QW] Z. Qin, W. Wang, Hilbert schemes of points on the minimal resolution and soliton equations, Contemp. Math. 442 (2007), 435-462. MR2372578 (2009b:14008)

[R] Z. Ran, Semiregularity, obstructions and deformations of Hodge classes, Ann. Scuola Norm. Sup. Pisa Cl. Sci. (4) 28 (1999), no. 4, 809-820. MR.1760539 (2001g:14014)

Department of Mathematics, Massachusetts Institute of Technology, Cambridge, MASSACHUSETTS 02139

E-mail address: dmaulik@math.mit.edu

Department of Mathematics, Princeton University, Princeton, New Jersey 08544

E-mail address: oblomkov@math.princeton.edu 